\title{
REAL-TIME DETECTION OF STORAGE COVERT CHANNELS
}

\author{
BY \\ THOMAS A. V. SATTOLO \\ A thesis submitted to the Faculty of Graduate and Postdoctoral Affairs \\ in partial fulfillment of the requirements for the degree of \\ Master of Applied Science \\ in \\ Electrical and Computer Engineering \\ Carleton University \\ Ottawa, Ontario, Canada \\ (C) 2021
}

Thomas A. V. Sattolo 


\section{Abstract}

Covert channnels are a class of techniques for hiding the presence of communication between parties. In the context of cybersecurity, covert channels can be used by attackers to evade detection and to exfiltrate sensitive data. In so doing, they create a need for effective detection techniques for the use of covert channels.

In this thesis, we present the conception, design and implementation of a system for detecting covert messages stored in the headers of network protocols in real time. We start by identifying statistical tests that can distinguish network traffic containing certain types of covert channels with high accuracy. We then leverage that information to build a system that analyses network traffic by tapping ethernet cables in order to detect the use of covert channels with very low latency. 


\section{Acknowledgements}

First of all, I would like to thank Prof. Jason Jaskolka for diligently supervising my work on this thesis. Without his steady guidance and frequent assistance this project surely would not have come to fruition as it has.

Furthermore, I would like to thank my family and for their love and kindness throughout these past two years. 


\section{Contents}

Abstract $\quad$ ii

Acknowledgements $\quad$ iii

Contents viii

List of Tables $\quad$ x

List of Figures $\quad$ xiii

List of Abbreviations $\quad$ xiv

1 Introduction 1

1.1 General Context. . . . . . . . . . . . . . . . . 1

1.2 Motivation . . . . . . . . . . . . . . . . . . 3

1.3 Problem Statement . . . . . . . . . . . . . . . . . 5

1.4 Contributions ........................ 6 


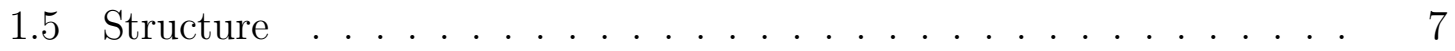

2 Background and State of the Art 9

2.1 Constructing Storage Covert Channels . . . . . . . . . . . . . 9

2.2 Overview of Covert Channel Detection . . . . . . . . . . . . . . 10

2.3 Key Works . . . . . . . . . . . . . . . . . . . . . 12

2.3.1 BroCCaDe.......................... 12

2.3.2 Covert Channel Detection Random Forest . . . . . . . . . . . 15

2.4 Real-time Covert Channel Detection . . . . . . . . . . . . . 16

2.5 Concluding Remarks . . . . . . . . . . . . . . . . . . . 17

$\begin{array}{lll}3 & \text { Statistical Tests } & 18\end{array}$

3.1 Key Statistical Concepts . . . . . . . . . . . . . . . . . . . 19

3.2 Test Classification . . . . . . . . . . . . . . . . . 22

3.2 .1 Complexity Tests . . . . . . . . . . . . . . . 23

3.2.2 Distributional Tests . . . . . . . . . . . . . . . . . . 24

3.3 Test Descriptions . . . . . . . . . . . . . . . 26

3.3.1 Complexity Tests . . . . . . . . . . . . 26

3.3.2 Distributional Tests . . . . . . . . . . . . . . . 35

4 Experiment $\quad 43$

4.1 Tests Used in the Experiment . . . . . . . . . . . . . . . 44 
4.2 Setup and Approach . . . . . . . . . . . . . . . . 44

4.2 .1 Building the Dataset . . . . . . . . . . . . . . . . . 44

4.2 .2 Conducting the Experiments . . . . . . . . . . . . . . 47

4.3 Results . . . . . . . . . . . . . . . . . . . . . . . . 48

4.3.1 Results for Tests Used in Isolation . . . . . . . . . . . . . . 48

4.3.2 Results for Tests Used in Combination . . . . . . . . . . . 54

4.3 .3 Logistic Regression Detector . . . . . . . . . . . . . . . 56

4.3.4 Repeating the Experiment for TCP ISN $\ldots \ldots \ldots$

4.3.5 Repeating the Experiment for IP TTL . . . . . . . . 66

4.4 Discussion . . . . . . . . . . . . . . . . . . . . . . . . . . . . . . . 72

$\begin{array}{lll}5 & \text { Implementation } & 73\end{array}$

5.1 Non-portable Components . . . . . . . . . . . . . . . . . . 74

$5.1 .1 \quad$ Board . . . . . . . . . . . . . . . . . 75

$5.1 .2 \quad$ Ethernet Core . . . . . . . . . . . . . . . . . . . . . 75

$5.1 .3 \quad$ Nios II Processor . . . . . . . . . . . . . . . . . . . . . . 77

5.1 .4 Phase Locked Loop . . . . . . . . . . . . . . . . . . . . 77

5.1.5 Top-level Module . . . . . . . . . . . . . . . . . . . . . 77

$5.2 \quad$ Software . . . . . . . . . . . . . . . . . . . 78

5.3 Hardware . . . . . . . . . . . . . . . . . . . . . . . . . . . . . . . . 79 
5.3.1 SEACCOW Internal . . . . . . . . . . . . . . . . . 79

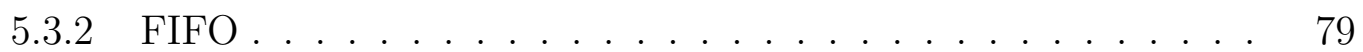

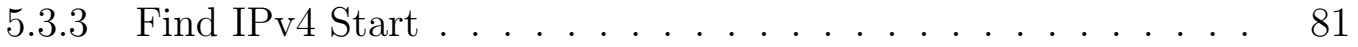

5.3.4 Get Field . . . . . . . . . . . . . . . . . . . . . . . . 81

5.3.5 Count Words . . . . . . . . . . . . . . . 82

5.3.6 Repetition .................... 83

5.3.7 Windowing ................... 85

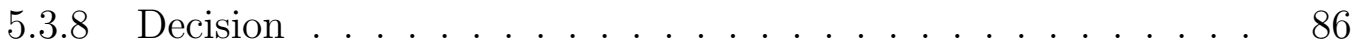

5.3.9 Repetition Test Bench . . . . . . . . . . . . . . . . 89

5.3.10 SEACCOW Internal Test Bench . . . . . . . . . . . . . . . 90

5.4 Performance . . . . . . . . . . . . . . . . . . . . . . . 90

5.4.1 Accuracy ................................ 90

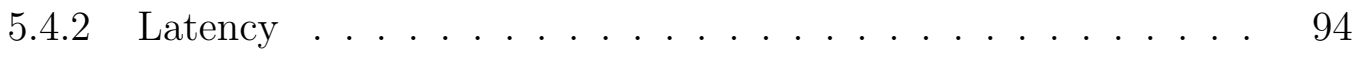

5.5 Concluding Remarks . . . . . . . . . . . . . . . . . . . 94

6 Conclusion $\quad 95$

6.1 Further Work . . . . . . . . . . . . . . . . 96

6.1.1 More Complex Covert Channels . . . . . . . . . . . . . . 96

6.1.2 Countermeasures To Evade Detection . . . . . . . . . . . . . 97

6.1.3 Long Term Memory . . . . . . . . . . . . . . . . . . . 97 
6.1.4 Generic Network Traffic Analysis . . . . . . . . . . . . . . . 97

6.1.5 More Sophisticated Test Combination . . . . . . . . . . . . . . 98

6.2 Final Remarks . . . . . . . . . . . . . . . . . . . . . . . . 98

$\begin{array}{ll}\text { A Covert Message } & 99\end{array}$

B Code Repositories 101

$\begin{array}{ll}\text { Bibliography } & 102\end{array}$ 


\section{List of Tables}

3.1 Sample Values for First-Order Entropy Demonstration . . . . . . . . 31

3.2 Example Sequences for Distributional Tests . . . . . . . . . . . 37

4.1 Tests Used in the Experiment . . . . . . . . . . . . . . . . 44

4.2 Effect Sizes for 256-byte messages in an IP ID covert channel . . . . . 49

4.3 Effect Sizes for 16-byte messages in an IP ID covert channel . . . . . 49

4.4 Effect Sizes for 1-byte messages in an IP ID covert channel . . . . . . 50

4.5 Correlation for 1-bit traces of 256-byte messages in an IP ID covert channel .......................... 55

4.6 Correlation for 1-bit traces of 16-byte messages in an IP ID covert

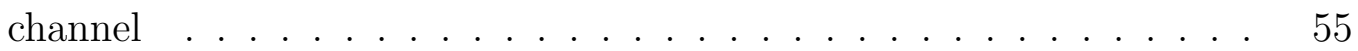

4.7 Detector accuracy for IP ID covert channel for various message sizes . 57

4.8 Detector accuracy for TCP ISN covert channel for various message sizes 61

4.9 Effect Sizes for 256-byte messages in a TCP ISN covert channel . . . 61

4.10 Effect Sizes for 16-byte messages in a TCP ISN covert channel . . . . 62

4.11 Effect Sizes for 1-byte messages in a TCP ISN covert channel . . . . . 62 
4.12 Detector accuracy for IP TTL covert channel for various message sizes 68

4.13 Effect Sizes for 256-byte messages in an IP TTL covert channel . . . 68

4.14 Effect Sizes for 16-byte messages in an IP TTL covert channel . . . . 69

4.15 Effect Sizes for 1-byte messages in an IP TTL covert channel . . . . . 69

4.16 Detector accuracy for IP TTL covert channel with 8-bit traces for various message sizes . . . . . . . . . . . . . . . . 70

5.1 SEACCOW Confusion Matrix . . . . . . . . . . . . . 91

5.2 SEACCOW Confusion Matrix with Class Imbalance . . . . . . . . . . 93

5.3 SEACCOW Confusion Matrix with Sub-optimal Threshold . . . . . . 93 


\section{List of Figures}

4.1 LZMA Effect Sizes for IP ID . . . . . . . . . . . . . . 51

$4.2 \quad$ LZ77 Effect Sizes for IP ID . . . . . . . . . . . . . . . . . . 51

4.3 LZ78 Effect Sizes for IP ID . . . . . . . . . . . . . . . . . . 52

4.4 LZC Effect Sizes for IP ID . . . . . . . . . . . . . . . . . 52

4.5 FOE Effect Sizes for IP ID . . . . . . . . . . . . . . . . 52

4.6 CCE Effect Sizes for IP ID . . . . . . . . . . . . . . . . . . . 52

4.7 REP Effect Sizes for IP ID . . . . . . . . . . . . . . . . . . 52

4.8 COV Effect Sizes for IP ID . . . . . . . . . . . . . . . . 52

4.9 KS Effect Sizes for IP ID . . . . . . . . . . . . . . . . 53

4.10 WCX Effect Sizes for IP ID . . . . . . . . . . . . . . . 53

4.11 SPR Effect Sizes for IP ID . . . . . . . . . . . . . . . . 53

4.12 REG Effect Sizes for IP ID . . . . . . . . . . . . . . . . . 53

4.13 256-byte Message REP-LZC Detector Probability Estimates . . . . . 58

4.14 64-byte Message REP-LZC Detector Probability Estimates . . . . . . 59 
4.15 16-byte Message REP-LZC Detector Probability Estimates . . . . . . 59

4.16 4-byte Message REP-LZC Detector Probability Estimates . . . . . . . 60

4.17 LZMA Effect Sizes for TCP ISN . . . . . . . . . . . . . . . 63

4.18 LZ77 Effect Sizes for TCP ISN . . . . . . . . . . . . . 63

4.19 LZ78 Effect Sizes for TCP ISN . . . . . . . . . . . . . . 63

4.20 LZC Effect Sizes for TCP ISN . . . . . . . . . . . . . . . 63

4.21 FOE Effect Sizes for TCP ISN . . . . . . . . . . . . . . . . . . 64

4.22 CCE Effect Sizes for TCP ISN . . . . . . . . . . . . . . . . 64

4.23 REP Effect Sizes for TCP ISN . . . . . . . . . . . . . . . . . . 64

4.24 COV Effect Sizes for TCP ISN . . . . . . . . . . . . . . . 64

4.25 KS Effect Sizes for TCP ISN . . . . . . . . . . . . . . . . . 64

4.26 WCX Effect Sizes for TCP ISN . . . . . . . . . . . . . . . 64

4.27 SPR Effect Sizes for TCP ISN . . . . . . . . . . . . . 65

4.28 REG Effect Sizes for TCP ISN . . . . . . . . . . . . . . . . . 65

4.29 LZMA Effect Sizes for IP TTL . . . . . . . . . . . . . . . . 70

4.30 LZ77 Effect Sizes for IP TTL . . . . . . . . . . . . . . 70

4.31 LZ78 Effect Sizes for IP TTL . . . . . . . . . . . . . . . . 70

4.32 LZC Effect Sizes for IP TTL . . . . . . . . . . . . . . . 70

4.33 FOE Effect Sizes for IP TTL . . . . . . . . . . . . . . . . 71

4.34 CCE Effect Sizes for IP TTL . . . . . . . . . . . . . . . 71 
4.35 REP Effect Sizes for IP TTL . . . . . . . . . . . . . . . . 71

4.36 COV Effect Sizes for IP TTL . . . . . . . . . . . . . . 71

4.37 KS Effect Sizes for IP TTL . . . . . . . . . . . . . . . . . 71

4.38 WCX Effect Sizes for IP TTL . . . . . . . . . . . . . . . . 71

4.39 SPR Effect Sizes for IP TTL . . . . . . . . . . . . . . . . . 72

4.40 REG Effect Sizes for IP TTL . . . . . . . . . . . . . . . . 72

5.1 DE2-115 Development Board . . . . . . . . . . . . 76

5.2 Block Diagram of Internal Modules . . . . . . . . . . . . . . . . . 80

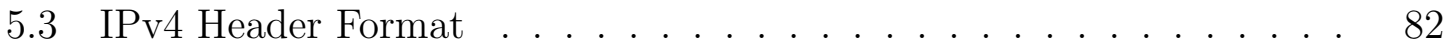




\section{List of Abbreviations}

CRC Cyclic Redundancy Check.

DNS Domain Name System.

FIFO First In First Out.

FPGA Field Programmable Gate Array.

HTTP Hypertext Transfer Protocol.

ICMP Internet Control Message Protocol.

ID Identification.

IP Internet Protocol.

IPv4 Internet Protocol Version 4.

ISN Initial Sequence Number.

MAC Media Access Control.

SEACCOW Security Evaluation and Assurance Covert Channel Online Warden.

TCP Transaction Control Protocol.

TTL Time-to-Live.

VLAN Virtual Local Area Network. 


\section{Chapter 1}

\section{Introduction}

This chapter introduces the concept of covert channels that will be the primary focus of this document. It begins with some basic definitions and categorizations in Section 1.1 followed by an account of the broad purpose in Section 1.2. Narrower goals are detailed in Section 1.3 and Section 1.4 briefly summarizes the results. The structure of this document is explained in Section 1.5.

\section{$1.1 \quad$ General Context}

A covert channel is a means of communication whose existence is hidden. More precisely, they were defined by Lampson as channels "not intended for information transfer at all" [Lam73]. This is a step beyond making a message indecipherable which is typically achieved using encryption. By making use of a covert channel one seeks not to obscure what message is being sent, but the very fact that a message is being sent. 
A technique such as this has no inherent moral character: it can by used maliciously or benevolently. An example of the latter could be to enable communication between two parties in a situation where the existence of any relationship would be sufficient to raise scrutiny. That said, the focus of this document will be on malicious uses of covert channels and the exfiltration of sensitive data as part of a data breach will be taken as the archetypal example of this.

The covert channels that exist in computer systems can be categorized in many ways, but two are particularly relevant to this thesis. The first of these binary classifications was proposed in [Jas10]. It involves the original purpose of the structures used to build the covert channel and the classes are as follows:

Protocol-based Covert Channels: A protocol-based covert channel is any covert channel that is built by abusing a formal communications protocol. An example of this would be sending a message using crafted TCP Initial Sequence Numbers; the message could be transmitted one bit at a time with an even sequence number indicating a ' 0 ' and an odd number indicating a ' 1 '.

Environment-based Covert Channels: Any covert covert channel that is not protocol based is environment based. These can exist when two or more agents have access to some of the same computing resources. An example of this would be sending a message by acquiring a file lock at specific times; a receiver could try to acquire a lock at constant time intervals (e.g. every minute) and infer a ' 1 ' if the lock is unavailable because it has already been acquired by the sender and a ' 0 ' otherwise. 
The other dichotomy concerns the dimension used to communicate the covert information. It is drawn from [US 85], the so-called 'Orange Book'.

Timing Covert Channels: A timing covert channel is one in which the recipient must infer a message from the timing of events. An example of this would be sending a message by varying the time interval between consecutive IP packets that are sent; a long delay-greater than some constant threshold-between packets could be used to indicate a ' 1 ' while a short time gap would indicate a ' 0 '.

Storage Covert Channels: For a storage covert channel the message is containedpossibly after being subjected to transformation (e.g. encryption, XOR) - in a location readable by recipient. A example of this would be sending a message using crafted TCP Initial Sequence Numbers. Note that this is the same example as was used for protocol-based covert channels, thus highlighting that a covert channel can fall under both categories.

Both these methods of categorization are binary i.e. each method divides covert channels into two classes. As such, these categorizations give rise to 4 classes of covert channels, including protocol-based storage covert channels which will be the particular focus of this project.

\subsection{Motivation}

Maintaining the confidentiality of user data is a serious and growing problem in the present information age. A study of the impact of data breaches was performed by 
the Ponemon Institute in 2018 [Ins18]. They found that, globally, the probability that an organization will suffer a data breach is increasing year over year. Moreover, the cost of each of these breaches is nothing to scoff at; they estimate that each data breach costs an average of 3.86 million dollars. At present, many of these breaches are caused by reasonably tractable problems such as system glitches and many others are presumably the work of an unsophisticated attacker who could have been stopped by security best-practices. However, even a well-prepared organization is likely to be vulnerable to an attacker using a covert channel to exfiltrate sensitive information.

The response to a data breach cannot start until it is detected by an organization's security controls and covert channels can be used to bypass these security controls. In so doing, they can amplify the impact of a data breach. Security-concious organizations generally monitor their networks for suspicious traffic and this will often be the way a breach is first noticed. However, the effect of covert channels is to allow attackers to move their communication outside of what will typically be monitored, thus delaying detection or even preventing it altogether. With attackers given more time to act, the amount of sensitive data whose confidentiality will be lost is sure to increase. By using covert channels to circumvent security controls attackers effectively increase the attack surface of a system.

This section has so far been focused on the value of detection, and that theme will continue throughout this thesis, but it would be remiss not to mention another important way to mitigate the problem of covert channels: a system can be designed such that covert channels simply do not exist. Restricting access to shared resourcesincluding, importantly the networking stack and the precise time - can go a long way 
towards preventing covert channels. Unless a resource is writeable by a hypothetical sender and readable by a hypothetical receiver, it cannot be used as a covert channel. This is known as the potential for communication condition [JKZ12]. Designing such a system would be simple enough, but it would come at great cost to the system's functionality. Programming and using a computer system where the only shared resources are those explicitly meant for communication would obviously be difficult and inefficient, which reaffirms the importance of detection.

\subsection{Problem Statement}

As mentioned in Section 1.1, the covert channels that exist in computer systems divide quite naturally into two groups, those that use timing and those that use storage. At present, more work is being done on the detection of timing covert channels. This presents an opportunity to do work evaluating the transferability of detection techniques used for timing covert channels to storage covert channels and hopefully elucidate some principles about what kind of tests can systematically uncover information covertly stored in the fields of a network protocol.

Furthermore, an important trait affecting the value of a covert channel detector is its ability to operate in real-time. Operating in real-time means having a strict upper bound on how much computing time can be used. In the context of detecting protocolbased covert channels any time used to compute is adds to the latency of the network and this must be kept low enough so that the system remains usable. Practically speaking, a delay shorter than $1 \mathrm{~ms}$ is unlikely to be deplored, but a system with 
with a latency much shorter than that seems achievable. A low latency is quite simply a requirement in order for anything to be done to secure the confidentiality of the information whose covert transfer is being detected; if detection is not done in real-time then the receiver will already be in possession of the relevant information before a response can even begin. To be sure, slower detection mechanisms can be useful to guard against repeated attack, but reacting immediately is desirable because it is a prerequisite for progressing from detection to prevention of an ongoing attack. Ultimately, the goal of any security technology is to prevent successful attacks from occurring, and speeding up detection techniques so as to offer effective prevention is a key step along that road. The main limitation on a covert channel detector working in real time is the latency between information being sent and it being detected; thus when trying to detect protocol-based storage covert channels it is important to reduce the time needed to access the relevant data as much as possible.

The goal of this project is to design and build a detector for protocol-based storage covert channels that will report results with low-enough latency to operate in real time.

\subsection{Contributions}

The contributions of this thesis are twofold. The first of these is an analysis of the effectiveness of various statistical tests at identifying protocol-based storage covert 
channels which would be useful to anyone else studying the detection of covert channels. This work was published as a conference paper at the $35^{\text {th }}$ International Conference on ICT Systems Security and Privacy Protection. Much of that paper is repeated herein with minor edits. This includes, most importantly, Chapter 4 which duplicates the bulk of the paper, excluding the paper's Abstract, Introduction, Related Work, and list of tests, but featuring additional data and results. Furthermore, Section 2.2 is a condensed version of the paper's Related Work section and parts of the test descriptions in Section 3.3, usually the rationale for each test's inclusion, are repeated from the published list of tests.

The second major contribution is the architecture of covert channel detector built using a Field Programmable Gate Array (FPGA). The platform was chosen so as to minimize the latency between covert channel use and detection. This is a goal that future work on covert channels is likely to have and many parts of the system (e.g. modules for receiving Ethernet frames and extracting header fields) have very generally applicability. The system presented in Chapter 5 and the accompanying code (available at: gitlab.com/CyberSEA-Public/SEACCOW) would be a useful starting point for any protocol-based covert channel detector implemented in hardware.

\subsection{Structure}

The remainder of this thesis is organized as follows:

Chapter 2: Background and State of the Art A survey of precursory work on covert channel detection. 
Chapter 3: Statistical Tests A detailed characterization of the statistical tests included in the subsequent experiment, along with some hypothesizing about their performance.

Chapter 4: Experiment A thorough accounting of the experimentation that was performed to determine the best statistical tests for a real-time protocol-based storage covert channel detector.

Chapter 5: Implementation A detailed description of the covert channel detection system that was implemented in hardware.

Chapter 6: Conclusion Some concluding remarks. 


\section{Chapter 2}

\section{Background and State of the Art}

This chapter seeks to sketch the landscape of the covert channel detection research that influenced this document. It begins with a primer on the creation of storage covert channels in Section 2.1. Next is an overview of many relevant papers in Section 2.2 followed by more detailed examinations in Section 2.3 of two bodies of work that were particularly influential to the direction of this project. Finally, Section 2.4 provides some background on existing detection methods that, similarly to this project, aim explicitly to operate in real time.

\subsection{Constructing Storage Covert Channels}

The task of creating Storage Covert Channels is mostly finding a location to store information that will not be monitored by security controls nor interfere with the proper functioning of a system when modified. The information that one wants to 
covertly transmit is stored in network protocol header fields, replacing what was already there. Transformations of any sort can be applied to the data before being transmitted so long as they are reversible. One such reversible transformation that is of particular interest is encryption, the effect of which on covert channel detection is studied in Chapter 4.

Gunadi and Zander [GZ17b] make a distinction between a Value Modulation pattern and a Reserved/Unused pattern. In the former, some values of the header field are taken to correspond to one of a set of symbols, e.g., small numbers are interpreted as logical zeroes; for the latter the content of the message is directly written to the header fields.

As for finding storage locations, many options are catalogued in [SK06]. They focus on storage locations in network protocol headers, i.e. on locations that would create protocol-based covert channels. These storage locations are fields in the IP, TCP, ICMP, HTTP and DNS headers.

\subsection{Overview of Covert Channel Detection}

When identifying the existence of covert channels in computer systems, particularly those which use network protocols as covert message carriers, anomaly detection has been among the most popular detection mechanisms. Anomaly detection refers to the detection of patterns in a given data set that do not conform to what is considered normal behaviour. Anomaly detection techniques are usually used in conjunction with machine learning and statistical approaches. 
As previously indicated, much of the existing work on detecting covert channels has focused on timing covert channels. Cabuk et al. [CBS04, CBS09] and Gianvecchio and Wang [GW10] proposed a variety of statistical tests focussed on detecting timing covert channels. Naik et al. [NBSD12] proposed an entropy approach for detecting timing channels. Similarly, Crespi et al. [CCG13] studied different statistical anomaly detection methods, commonly used in network traffic analysis, to detect timing covert channels.

While there has been a focus on timing channels, approaches for detecting storage covert channels have also been proposed. Sohn et al. [SSM03] proposed an offline covert channel detection technique using a support vector machine to search for anomalies in the header fields of network packets. A similar technique was proposed by Tumoian and Anikeev [TA05] involving the interception of all TCP traffic and a model of the initial sequence number generation. The idea uses a neural network to create a model using only the intercepted TCP traffic without any knowledge of the data generation algorithm in an attempt to identify anomalies in the initial sequence numbers of the intercepted TCP segments. Xing et al. [XKC20] opt to focus on mitigation rather than detection and propose NetWarden to combat the use of TCP covert channels by leveraging the programmable data planes of modern network switches. Berk et al. [GBC05, BGC05] investigated a methodology for detecting such channels based on a statistical measure of how well the capacity of a given channel is achieved. Jadhav and Kattimani [JK11] proposed a statistical detection method involving the capture of TCP segments from active network streams and analyzing the covert channel vulnerable fields of TCP headers. Zhai et al. [ZLD10] proposed a method based on a TCP Markov model and the Kullback-Leibler divergence [KL51] 
to verify the existence of anomalies in the TCP Flags field. Zhao and Shi [ZS10] aimed to detect the existence of covert information embedded in TCP Initial Sequence Numbers using phase-space reconstruction to represent the dynamic nature of initial sequence numbers by building a four-dimensional space of the one dimensional initial sequence numbers.

\section{$2.3 \quad$ Key Works}

Whereas the preceding section summarized many papers in a few paragraphs, this one will provide a much deeper analysis of two projects - one individual paper and a series of papers - that influenced this thesis a great deal.

\subsubsection{BroCCaDe}

In 2017, Gunadi and Zander embarked on a project to extend the Zeek (formerly Bro) network security monitoring tool to detect covert channels. They named their framework 'Bro Covert Channel Detection' or BroCCaDe for short; Bro was renamed to Zeek at roughly this time. Nevertheless, they published their work in a series of four reports covering the Scope and Background [GZ17b], Design and Implementation [GZ17a], Extension [GZ17c] (i.e. they explain how others should extend their framework), and Performance [GZ18], respectively.

The scope of BroCCaDe is limited by the abilities of Zeek. Firstly, being a network monitoring tool, Zeek is only useful for dealing with protocol-based covert channels; 
it also does not easily handle link-layer and physical-layers protocols, thus covert channels at these layers are out of scope. Besides the scope limitations imposed by Zeek, the authors also restrict themselves to a set of Covert Channel patterns and a set of Analysis metrics.

The patterns considered are as follows [GZ17b]:

Size Modulation In which the size of a protocol data unit (e.g., an IP packet or a TCP segment) is modulated to communicate a message. This is a storage covert channel.

Value Modulation In which the value of a header field is modulated. This is a storage covert channel.

Reserved/Unused In which the message is stored in unused header fields. This is different from value modulation in that for the value modulation pattern the value of a field is alternated between two or more legitimate values for the field to take and the pattern is what communicates message whereas in the reserved/unused pattern the contents of the message is directly stored where the header field should be. Once again, this is a storage covert channel.

Inter-arrival Pattern In which the message is encoded into the length of interpacket gaps. This is a timing covert channel.

Rate Pattern In which the message is encoded into the rate of packet arrival. This is a timing covert channel. 
The analysis metrics are as follows [GZ17b] (see Section 3.3 for more on these techniques):

Kolmogorov-Smirnov Test Given random variables $X$ and $Y$,

$$
K S(X, Y)=\sup _{x}|P(X<x)-P(Y<x)|
$$

Entropy Given random variables $X$ and, $H(X)=-\sum_{i} P\left(X=x_{i}\right) \log P\left(X=x_{i}\right)$.

Corrected Conditional Entropy A fairly complex measure designed to correct for issues arising from a limited number of available samples when calculating entropy.

Multi Modal Analysis Using a histogram of random variable $X$, calculate $M(X)=$ $\frac{\sum n_{i}^{2}}{\max \left(n_{i}^{2}\right)}$. Not covered in Section 3.3.

Autocorrelation The correlation between a sequence and a shifted version of itself.

Regularity A measure of the changes in the standard deviation of a sequence.

BroCCaDe's design is characterized by two parameters that control the way network communications are sampled for testing by the analysis metrics. These are the window size and the step size [GZ17a]. The window size is the number of contiguous packets used for each run of analysis and the step size is the number of packets between the start of one run of analysis and the start of the next. The step size can be larger or smaller than the window size. A step size larger than the window size implies that some packets will be totally ignored whereas the reverse implies that some packets are analysed multiple times. Both parameters can be tuned based on the pattern, the analysis metric and the performance requirements. 
The ability of BroCCaDe to detect covert channels is quite good; it can achieve a true positive rate of $98 \%$ and a false negative rate of less than $1 \%$ [GZ18]. It does this with only modest CPU and memory usage. However, what it does not do is real-time analysis. It is evaluated on stored traces and the authors make no attempt to analyse packets as they pass through the network and produce results with low latency.

\subsubsection{Covert Channel Detection Random Forest}

Li et al. published a paper in 2017 about using a random forest classifier to detect timing covert channels [LZCF17]. This paper was particularly important because random forest classifiers work by aggregating a variety of different statistical tests and the paper assesses the usefulness of many tests for detecting timing covert channels. Since evaluating statistical tests is among the goals of this thesis - implementing extraneous tests on an FPGA would be time-consuming - this paper was an excellent source of potential tests to evaluate. To be clear, Li et al. worked exclusively with timing covert channels whereas this thesis is focused on storage covert channels. Nonetheless, exploring the difference between which tests work for each type of covert channel is of interest in and of itself.

Besides analysing tests, the authors also evaluated their random forest classifier and found that it was not particularly effective. A low base rate of positive examples (i.e. packets containing covert information) led to a rate of false positives that was consistently above $10 \%$. 


\subsection{Real-time Covert Channel Detection}

An important way in which this thesis differentiates itself from previous work is by its focus on real-time detection of covert channels. That said, important work has been done to detect covert channels in real time and some of that work is highlighted in this section.

Liu et al. [LCY11] proposed a system in which covert channels are detected by using a virtual machine to mimic the behaviour of a system. Discrepancies between the behaviour of the original system and the replica are used to detect the use of covert channels. A 2015 paper by two of the same authors (and one other) $\left[\mathrm{LCW}^{+} 15\right]$ applies a similar approach to the real-time detection of covert channels in software-defined networking.

Gegan et al. [GAOG16] take a similar approach to this thesis in that they use known tests and build a detector that manages to operate in real-time by using specialized hardware. However, they use GPUs rather than FPGAs and try to detect timing covert channels rather than storage covert channels. Moreover, they restrict their study to entropy-based tests, namely first-order entropy and corrected conditional entropy.

Finally, Rezaei et al. [RHS17] use a subset of the non-parametric statistical tests used by Fu et al. [LZCF17] (see Subsection 2.3.2) to construct a real-time detector for covert timing channels. The tests are used separately (i.e. they are not combined using a random forest or the like) and achieve accuracies over $90 \%$ with only a few milliseconds of computational overhead. 


\subsection{Concluding Remarks}

This chapter begins with some information of how covert channels are constructed in order to provide context on the topic. However, the main goal is to review the available research that has similar goals to this thesis. First, several papers that present detection techniques are summarized, then other projects that align more closely with this project motivations - either by focusing on broad detection systems featuring a variety of statistical tests or by aiming to report results in real time - are explained in greater detail. 


\section{Chapter 3}

\section{Statistical Tests}

The task of detecting storage covert channels requires statistical tests that will differentiate channels containing covert information from those that do not. There are many potential such tests that have been devised and many of them have been successfully used to detect timing covert channels but only a few have been applied to storage covert channels. This chapter covers the background that a reader should know about the statistical tests evaluated in this thesis. Section 3.1 provides a review of some key concepts. Section 3.2 follows with some theoretical predictions about which tests ought to perform best. Section 3.3 ends the chapter with a description of each test along with the rationale for its inclusion in the experiment chronicled in Chapter 4 . 


\subsection{Key Statistical Concepts}

The following definitions of key statistical concepts are taken from [Dod08]. These concepts are needed to understand the various statistical tests for covert channel detection that are considered in this work.

Random Variable: A variable whose value is determined by the result of a random experiment is called a random variable. [...] An experiment in which the outcome is not predictable in advance is called a random experiment. A random experiment can be characterized as follows:

1. It is possible to describe the set of all possible results (called the sample space of the random experiment).

2. It is not possible to predict the result with certainty.

3. It is possible to associate each possible outcome to a probability of appearing.

Expected Value: The expected value of a random variable is the weighted mean of the values that the random variable can take, where the weight is the probability that a particular value is taken by the random variable.

Standard Deviation: The standard deviation is a measure of dispersion. It corresponds to the positive square root of the variance, where the variance is the mean of the squared deviations of each observation with respect to the mean of the set of observations. 
Probability Mass Function: The probability [mass] function of a discrete random variable is a function that associates each value of this random variable to its probability.

Conditional Probability: The probability of an event given that another event is known to have occurred. The conditional probability is denoted $P(A \mid B)$, which is read as the "probability of A conditioned by B."

Covariance: The covariance between two random variables $\mathrm{X}$ and $\mathrm{Y}$ is the measure of how much two random variables vary together. If $\mathrm{X}$ and $\mathrm{Y}$ are independent random variables, the covariance of $\mathrm{X}$ and $\mathrm{Y}$ is zero. The converse, however, is not true.

Correlation: The simple correlation coefficient is a measure of the strength of the linear relation between two random variables. The correlation coefficient can take values that occur in the interval $[-1,1]$. The two extreme values of this interval represent a perfectly linear relation between the variables, "positive" in the first case and "negative in the other". The value 0 (zero) implies the absence of a linear relation.

Two final concepts remain that were not adequately defined in [Dod08]:

Effect size: Effect size is a quantification of the difference between two groups. Consider two groups $A$ and $B$ each with some distribution. The effect size is the number of pooled standard deviations between the mean of $A$ and the mean of $B$. The pooled standard deviation is the square root of the mean of the variances of $A$ and $B$. 
Kolmogorov Complexity: Kolmogorov complexity is one of many mathematical constructs that can be used to detect covert channels. The process of creating covert channels can be viewed as taking data that is typically random and interfering with it to make it not random. The usefulness of this view is predicated on having a precise and functional definition of randomness; and this is what the concept of Kolmogorov Complexity seeks to provide.

In some sense, all strings are equally random. When drawing from the set of all binary strings of length 32 , every outcome is equally likely whether it is:

\section{11111111111111110000000000000000, or}

2. $10110100101010010011001101000111^{1}$

However, there is an obvious intuitive sense in which the binary string labelled 2 is random and the one labelled 1 is not. This raises the question of how to define the randomness of a string in a precise and useful way.

Kolmogorov Complexity is a concept that seeks to answer this question. In essence, the Kolmogorov Complexity of a string $s$ is defined as the length of the shortest description than can uniquely specify $s$ [For01]. This description can be in any language or formal system and the strings whose Kolmogorov complexity is closer to their actual length are more random than others. Note that simply describing the strings above in plain English gives a good idea of how long a unique description of each would have to be: 'Sixteen ones and Sixteen zeros' is a lot shorter than 'A one, a zero, two ones, a zero, a one, two zeros, a one, a zero, a one, a zero etc.'

\footnotetext{
${ }^{1}$ True random number generated with 'head /dev/random -c 4 | xxd -b'
} 
Another way to view Kolmogorov Complexity is as the length of the optimal way to compress any string. Seen this way, it is unsurprising that the Kolmogorov Complexity of a string is not computable [CAC95]. That said, it is still a very useful concept not least because it can be approximated by a string's compression ratio ${ }^{2}$ with widely-available lossless compression algorithms $\left[\mathrm{LCL}^{+} 03\right]$.

\subsection{Test Classification}

Statistical tests previously used to detect timing covert channels can be classified into two general categories: Complexity Tests and Distributional Tests.

Broadly speaking, complexity tests rely on differences in the information content of covert channel traffic as opposed to normal traffic, whereas distributional tests work by analyzing the difference in the empirical probability distributions of covert and normal traffic. This distinction is important because each category of tests is viewed differently in its applicability to storage covert channels.

All these tests are functions that take as input a sequence of network header fields that may contain covert information and output a single number. Some tests also require a comparison sequence of header fields known not to contain covert information. For all tests, the key consideration is how the presence of covert information in the input affects the output. A test is effective if and only if the output value is reliably different when a sequence of headers fields has been used as a covert channel. In this case, a threshold can be established such that the vast majority of sequences containing

\footnotetext{
${ }^{2}$ original size over compressed size
} 
covert information will fall on one side and those lacking it will fall on the other.

\subsubsection{Complexity Tests}

Complexity tests attempt to measure the extent to which a stream of data is random or predictable and they work if the information transmitted via a covert channel is systematically different from normal network traffic in this way. This works with timing covert channels [GW10, CBS09, GZ18], and the concept should extend to storage covert channels, with some caveats. Firstly, it is not necessarily the case that traffic becomes less random when used as a covert channel ${ }^{3}$. This ought to be the case with some fields of network packet headers such as TCP Sequence numbers and IP Identification numbers that can be expected to be random under normal circumstances, but not with others such as IP Flags that would usually just be the same for most packets. The latter, of course, become more random once they have covert information stored in them. It should be possible to determine thresholdsones that are different for each field - that will separate normal traffic from that when a covert channel is being used.

There are many ways to measure the complexity of a stream of data and thus there are many potential complexity tests to consider. Nonetheless, they can be divided into two types: compressibility-based tests and entropy-based tests.

The compressibility of a stream of data can be used as measure of its

\footnotetext{
${ }^{3}$ This is the case with timing covert channels because normal inter-packet delays are essentially random and those of covert channels cluster at either of the values used as symbols in the communication.
} 
randomness - more compressible data is less random. At a limit, the size of a stream of data is equal to its Kolmogorov Complexity, implying that it cannot be compressed at all. Where it falls along this axis from very predictable and non-random to incompressible can be informative as to the likelihood that the stream is being used as a covert channel. A technique based on this principle was used by Cabuk et al. [CBS09] to detect timing covert channels. They examined a covert channel created using the arrival times of IP packets; messages were sent by alternating long and short delays between packets to represent binary digits. They found that the arrival time pattern of this traffic could be separated from normal traffic because it was more compressible than normal, unaltered traffic.

Entropy is a measure of the information contained in data. As such, it is the most direct tool to search for covert information. Both unused network protocol fields and the covert messages that people may send contain some information, but there is no particular reason for them to contain the same amount of information per byte sent. If these happen to differ, measuring the entropy of unused network protocol fields has the potential to reveal covert channels if they exist.

All of the complexity tests evaluated in our experiments are ultimately based on either compressibility or entropy.

\subsubsection{Distributional Tests}

Much of the work of statistical science is to quantify the extent to which two or more entities are different in a world of limited observations. Fundamentally, this is the 
same as the task of identifying a covert channel so it stands to reason that the huge body of work that exists in statistics would be useful for detecting covert channels. In the literature, several different statistical features - ones that have nothing to do with entropy or compressibility — of inter-packet delay data have been used successfully to detect timing covert channels [LZCF17, CBS04, GZ18].

However, there is reason to believe that the usefulness of these tests will not transfer to storage covert channels. Underlying the logic of these distributional tests is the assumption that the data provided to them is measuring something; they involve ordinal comparisons between the elements in putative covert channels. This makes sense for timing covert channels where each element is an inter-packet delay, measured in seconds, and ordering inter-packet delays is obviously meaningful. The same cannot be said of storage covert channels, where each element is just the data observed in a network protocol header field. These are not measuring anything, are dimensionless, and ordering them in any way is spurious.

In spite of this, the applicability of distributional tests to storage covert channels cannot be so easily dismissed. Distributional tests seem less likely than complexity tests to transfer to storage covert channels, but abstract reasoning is no match for experiment, so a range of distributional tests will be considered and evaluated in our study. 


\subsection{Test Descriptions}

In this section, we classify and describe the statistical tests that are considered for storage covert channel detection. We also provide a rationale for each test's inclusion in the experiment described in Chapter 4.

\subsubsection{Complexity Tests}

\section{Lempel-Ziv Complexity}

In a 1976 paper, Abraham Lempel and Jacob Ziv [LZ76] proposed a novel metric to assess the complexity of a string. Their method is explicitly aimed at creating a way of quantifying how "random-looking" [LZ76] a string is.

Given a string $S$ made up of characters from alphabet $A$, the number of steps needed to build $S$ starting from the empty string. With $S_{i}$ indicating the string at the beginning of the $i^{\text {th }}$ step, on each step one can do one of the following things:

1. Take a substring of $S_{i}$ and append to the end of $S_{i}$.

2. Take a suffix of $S_{i}$ (a substring that includes the end of $S_{i}$ ) and append any number of copies of it to $S_{i}$. The final copy can be partial, i.e. the number of copies can be fractional.

One can also add one arbitrary character from $A$ to the end of $S_{i}$ in the 
same step.

For example, given the binary string 001 one could make 0010100 in a single step by appending the substring 01 once fully to make 00101 , once partially to make 001010 and then adding a 0 to the end. For many strings, there will be many series of steps that could have been used to create it; the Lempel-Ziv complexity is the length of the shortest such series of steps.

The second option is the preceding list of step constituents can equivalently be described as appending a single substring to $S_{i}$, but where the set of substrings is updated continuously as one adds characters to $S_{i}$. For example, if $S_{i}$ starts off as 101 it is valid to append 010 in a single step because 01 is a suffix of 101 and as soon as the initial 0 of 01 is appended to $S_{i}$ it becomes 1010 which has 010 as a substring. This kind of behaviour ought to be quite familiar from a computer programming perspective. With strings stored as arrays of characters and each substring stored as a pointer to a location within the array and a length, this is the behaviour that would be exhibited by a program appending a substring one character at a time if it is given a length that extends past the end of the string.

Lempel-Ziv complexity is a precursor to work on compression. Seeing as the compression ratio of covert traffic is expected to be a useful test to detect covert channels, it is only natural to also consider this related idea that is meant specifically to be a complexity measure. 


\section{Lempel-Ziv 77 Compression}

Furthering their work on string complexity, Lempel and Ziv developed a compression algorithm that has come to be know as LZ77 after its authors and the year of its publication [ZL77]. As with any compression algorithm, the compression ratio produced by LZ77 will depend on the complexity of the string.

The compression procedure for LZ77 is a straightforward extension of the algorithm used to compute Lempel-Ziv complexity. Per the preceding section, this algorithm describes a string as a series of steps; the LZ77 compression procedure stores strings in terms of these steps. Each step can be represented as a pointer to a location in the existing string, a length, and a final character. The output of the LZ77 compression algorithm is not necessarily based on the shortest such sequence of steps because determining the optimal sequence is likely to be impractical. Limits are imposed on the substring length and its starting point's distance from the end of the string in order to ensure that the pointer and length can be stored as a constant number of bits.

For example, given the 13 character string 1011101110010 of which the first 10 characters 1011101110 have already been compressed, one could store the final 3 characters 010 as $\{4,2,1\}$ signifying a step taking a substring 2 characters long starting 4 characters from the end of the already compressed string (the last character is 0 characters from the end) followed by a 0 . 
LZ77 is among the simplest compression algorithms available. Since the overall performance (compression ratio) of the compression is not directly relevant to the purpose of our experiments - all that matters is the difference between how it compresses covert channel traffic versus innocent traffic - it is worthwhile to include a very simple compression algorithm like Lempel-Ziv 77 [ZL77] as a test in the experiment.

\section{Lempel-Ziv 78 Compression}

Lempel-Ziv 78 or LZ78 is an improvement over LZ77 that was published in the following year [ZL78]. The key change from LZ77 to LZ78 is that instead of storing a string as a series of substrings, one instead stores the string as a series of code words. Each code word has a predetermined length so this saves having to store a length value for each one. The set of available code words can be reconstructed by the decompression process from the code words it has already seen; put simply a new code word is added to the set every time the sequence of characters received is not a prefix (i.e. a substring including the beginning of a string which can be the whole string) of any previous code word. Note that as in LZ77, a single character is stored with each code word, allowing previously unseen patterns to be stored.

For example, given the 13 character string 10111011100110 of which the first 11 characters 10111011100 have already been compressed, the existing code words would be $\{1,0,11,10,111,00\}$. The final 3 characters 110 could be stored as $\{3,0\}$ signifying the third code word followed by a 0 .

Clearly, Lempel-Ziv 78 is quite similar to Lempel-Ziv 77 , as such the reasons it is 
included here are the same.

\section{Lempel-Ziv Markov Chain}

Lempel-Ziv Markov Chain compression or LZMA for short is a modern refinement of LZ78 and LZ77. As its name suggests, it makes use of Markov chains, which are a mathematical tool to describe the states of a system and the probability of a transition from any one state to any other. LZMA is very widely used as it is the underlying algorithm for the popular compression utility 7zip [Pav19]. As for

performance, LZMA offers a high compression ratio in exchange for relatively low compression speed [Col05]. The compression ratio is what is reported as the output of the test.

As with all the compression algorithms among the tests considered in our experiments, the rationale for studying LZMA to detect storage covert channels comes from [CBS09].

\section{First-Order Entropy}

For discrete quantities, the entropy of random variable $X$ is defined as follows:

$$
H(X)=-\sum_{i} p\left(x_{i}\right) \log _{2} p\left(x_{i}\right)
$$

in which $p(X)$ is the probability mass function of $X$. Using the base 2 logarithm means that the value $H(X)$ will be given in bits. When working with data sampled 
Table 3.1: Sample Values for First-Order Entropy Demonstration

\begin{tabular}{cc}
\hline Value & Occurrences \\
\hline 1 & 6 \\
2 & 5 \\
3 & 7 \\
4 & 2 \\
\hline
\end{tabular}

from an arbitrary source, one does not generally know the probability mass function of the underlying random variable, thus the entropy cannot be directly computed. Nonetheless, the probability mass function can be approximated from a histogram of the sampled data. Using this approximation to compute $H(X)$ with the equation above yields the first order entropy. For example, given a random variable $X$ uniformly distributed over the set $\{1,2,3,4\}$, over 20 samples one might see the results shown in Table 3.1.

In this case, the entropy based on the probability mass function would be computed as follows: 


$$
\begin{aligned}
H(X)= & -\sum_{i} p\left(x_{i}\right) \log _{2} p\left(x_{i}\right) \\
=- & \left(P(X=1) \log _{2} P(X=1)+P(X=2) \log _{2} P(X=2)\right. \\
& \left.+P(X=3) \log _{2} P(X=3)+P(X=4) \log _{2} P(X=4)\right) \\
= & -\left(\frac{1}{4} \log _{2} \frac{1}{4}+\frac{1}{4} \log _{2} \frac{1}{4}+\frac{1}{4} \log _{2} \frac{1}{4}+\frac{1}{4} \log _{2} \frac{1}{4}\right) \\
=- & \left(\frac{1}{4}(-2)+\frac{1}{4}(-2)+\frac{1}{4}(-2)+\frac{1}{4}(-2)\right) \\
= & -\left(-\frac{1}{2}-\frac{1}{2}-\frac{1}{2}-\frac{1}{2}\right) \\
= & -(-2) \\
= & 2
\end{aligned}
$$

On the other hand, the first-order entropy based on the sampled histogram would be computed as follows: 


$$
\begin{aligned}
H_{f}(X)=- & \sum_{i} p\left(x_{i}\right) \log _{2} p\left(x_{i}\right) \\
=- & \left(P(X=1) \log _{2} P(X=1)+P(X=2) \log _{2} P(X=2)\right. \\
& \left.+P(X=3) \log _{2} P(X=3)+P(X=4) \log _{2} P(X=4)\right) \\
=- & \left(\frac{6}{20} \log _{2} \frac{6}{20}+\frac{5}{20} \log _{2} \frac{5}{20}+\frac{7}{20} \log _{2} \frac{7}{20}+\frac{2}{20} \log _{2} \frac{2}{20}\right) \\
=- & \left(\frac{3}{10}(-1.737)+\frac{1}{4}(-2)+\frac{7}{20}(-1.515)+\frac{1}{10}(-3.322)\right) \\
= & -(-0.521-0.5-0.530-0.332) \\
= & -(-1.883) \\
= & 1.883
\end{aligned}
$$

This demonstrates the relationship between entropy and first-order entropy, showing that one is related to the other but that they are not generally the same. First-order entropy was used to detect timing covert channels in [LZCF17, GZ17a].

\section{Corrected Conditional Entropy}

As stated in the previous section, entropy is defined in terms of a probability mass function. This is equivalent to saying that the true entropy of a sequence can only be computed after it has been sampled infinitely many times since that is the only way to know the exact probability mass function. Originally developed in the field of biological cybernetics, Corrected Conditional Entropy is a alternative metric for the complexity of a sequence that aims to approximate the true entropy while remaining 
computable $\left[\mathrm{PBL}^{+} 98\right]$. It was imported into the field of covert channel detection by Gianvecchio and Wang [GW10].

Given a sequence $X$ of length $N$, the first step is to transform $X$ into a sequence of $N-L+1$ vectors of length $L$ in which each vector is composed of $L$ consecutive samples of $X$. For example, given $X=\{2,4,6,8,10,12\}$ and $L=3$ the transformed sequence would be:

$$
T(X, 3)=\{(2,4,6),(4,6,8),(6,8,10),(8,10,12)\}
$$

With this, one can define the first-order entropy of a transformed sequence:

$$
H^{\prime}(X, L)=H_{f}(T(X, L))
$$

The conditional entropy of a transformed sequence is given by:

$$
H^{\prime}(X, L \mid L-1)=H^{\prime}(X, L)-H^{\prime}(X, L-1)
$$

Finally, $U$ will be a function that takes a sequence and returns the fraction of unique values within that sequence. The Corrected Conditional Entropy is then defined as:

$$
C C E(X, L)=H^{\prime}(X, L \mid L-1)+U(T(X, L)) H_{f}(X)
$$

The Corrected Conditional Entropy can be computed for any value of $L \leq N$; the best estimate for the true entropy is given by the minimum Corrected Conditional Entropy value for any value of $L$. 


\section{Repetition}

The repetition rate is the fraction of values in a sequence that are repeated, i.e. they are equal to at least one other element. For example, given the sequence $X=$ $\langle 2,5,4,5,0,1,1,9,5,7\rangle$ the repetition rate would be $\frac{3}{10}=0.3$ because $X$ is 10 values long and has 3 values that are repeated, the second and third $5^{\prime} s$ and the second 1.

Although repetition might turn out to be a practical test, it functions largely as a sanity check. Other tests considered in our study should generally perform as well or better than such a simple and easily computable measure.

\subsubsection{Distributional Tests}

\section{Autocovariance}

Autocovariance is the covariance of a sequence with a shifted version of itself. It was used to detect timing-based covert channels by Gunadi and Zander [GZ17a], as well as Li et al. [LZCF17].

For a sequence $X$ of length $N$ with mean $\mu_{X}$, the autocovariance is given by Equa-

tion 3.8, where $X_{i}$ is the $i^{t h}$ element of $X, E(X)$ is the expected value and $S$ is the number of elements by which the sequence is shifted. 


$$
\begin{aligned}
A(X, S) & =\operatorname{cov}\left(X_{i} X_{i-S}\right) \\
& =E\left(\left(X_{i}-E\left(X_{i}\right)\right)\left(X_{i-S}-E\left(X_{i-S}\right)\right)\right) \\
& =\sum_{i=S+1}^{N}\left(X_{i}-\mu_{X}\right)\left(X_{i-S}-\mu_{X}\right)
\end{aligned}
$$

For example, given $X=\langle 5,10,8,16,14,28,26\rangle$ and $S=1$, one would have:

$$
\mu_{X}=E\left(X_{i}\right)=\frac{1}{N} \sum_{i=1}^{N} X_{i}=\frac{1}{7} 107=15.286
$$

And:

$$
\begin{aligned}
A(X, 1)= & \operatorname{cov}\left(X_{i} X_{i-1}\right) \\
= & E\left(\left(X_{i}-E\left(X_{i}\right)\right)\left(X_{i-1} E\left(X_{i-1}\right)\right)\right) \\
= & \sum_{i=S+1}^{N}\left(X_{i}-\mu_{X}\right)\left(X_{i-1}-\mu_{X}\right) \\
= & (10-15.286)(5-15.286)+(8-15.286)(10-15.286) \\
& \quad+(16-15.286)(8-15.286)+(14-15.286)(16-15.286) \\
& \quad+(28-15.286)(14-15.286)+(26-15.286)(28-15.286) \\
= & (-5.286)(-10.286)+(-7.286)(-5.286)+(0.714)(-7.286) \\
& \quad(-1.286)(0.714)+(12.714)(-1.286)+(10.714)(12.714) \\
= & 54.372+38.514-5.202-0.918-16.350+136.218 \\
= & 206.634
\end{aligned}
$$


Table 3.2: Example Sequences for Distributional Tests

\begin{tabular}{cccc}
\hline \multicolumn{2}{c}{ X } & \multicolumn{2}{c}{$\mathrm{Y}$} \\
\hline Rank & Value & Rank & Value \\
\hline 1 & 2 & 2 & 8 \\
2 & 7 & 1 & 6 \\
3 & 9 & 4 & 17 \\
4 & 12 & 3 & 14 \\
\hline
\end{tabular}

\section{Kolmogorov-Smirnov Test}

The Kolmogorov-Smirnov test is a widely used measure that tests whether or not two samples were drawn from sources with the same underlying probability distribution. It has also been used in [LZCF17, GZ17a] to detect timing covert channels. The formula for the Kolmogorov-Smirnov test is very simple, one just takes the maximum difference between the cumulative distribution functions as shown in Equation 3.11.

$$
K S(X, Y)=\sup _{x}|P(X<x)-P(Y<x)|
$$

The supremum function over $x, \sup _{x}$, simply returns the largest values of the expression it is given for any value of $x$.

Using the sequences from Table 3.2, the resulting Kolmogorov-Smirnov test value is: 


$$
\begin{aligned}
& K S(X, Y)=\sup _{x}|P(X<x)-P(Y<x)| \\
&=\sup \{|P(X<2)-P(Y<2)|,|P(X<6)-P(Y<6)|, \\
&|P(X<7)-P(Y<7)|,|P(X<8)-P(Y<8)|, \\
&|P(X<9)-P(Y<9)|,|P(X<12)-P(Y<12)|, \\
&|P(X<14)-P(Y<14)|,|P(X<17)-P(Y<17)| \\
&= \sup \left\{|0-0|,\left|\frac{1}{4}-0\right|,\left|\frac{1}{4}-\frac{1}{4}\right|,\left|\frac{1}{2}-\frac{1}{4}\right|,\right. \\
&\left.\left|\frac{1}{2}-\frac{1}{2}\right|,\left|\frac{3}{4}-\frac{1}{2}\right|,\left|1-\frac{1}{2}\right|,\left|1-\frac{3}{4}\right|\right\} \\
&= \sup \left\{0, \frac{1}{4}, 0, \frac{1}{4}, 0, \frac{1}{4}, \frac{1}{2}, \frac{1}{4}\right\} \\
&= \frac{1}{2} \quad
\end{aligned}
$$

\section{Spearman Correlation}

The Spearman Correlation is the correlation between the ranks of two sequences. It has been used to detect timing covert channels in [LZCF17]. The rank of a sequence is obtained by replacing each value in the sequence with the position it would take if the sequence were to be sorted, i.e. 1 replaces the smallest value, 2 replaces the second smallest and so on. $R(X)$ will be used to denote the rank of a sequence $X$.

Using the sequences from Table 3.2 the resulting Spearman Correlation test value is: 


$$
\begin{aligned}
\rho_{S}(X, Y) & =\frac{\operatorname{cov}(R(X), R(Y))}{\sigma_{R(X)} \sigma_{R(Y)}} \\
& =\frac{E((R(X)-E(R(X)))(R(Y)-E(R(Y))))}{\sigma_{R(X)} \sigma_{R(Y)}} \\
& =\frac{E\left(\left(R(X)-\mu_{R(X)}\right)\left(R(Y)-\mu_{R(Y)}\right)\right)}{\sigma_{R(X)} \sigma_{R(Y)}}
\end{aligned}
$$

Where:

$$
\mu_{R(X)}=\mu_{R(Y)}=E(R(Y))=\sum R(Y)=\frac{1}{4} 10=2.5
$$

And:

$$
\sigma_{R(X)}=\sigma_{R(Y)}=\sqrt{E\left(\left(R(Y)-\mu_{R(Y)}\right)^{2}\right)}=\sqrt{\frac{1}{4} 5}=1.12
$$

Which results in a Spearman Correlation of:

$$
\begin{aligned}
\rho_{S}(X, Y) & =\frac{\sum\left(R(X)-\mu_{R(X)}\right)\left(R(Y)-\mu_{R(Y)}\right)}{4 \sigma_{R(X)} \sigma_{R(Y)}} \\
& =\frac{(-1.5)(-0.5)+(-0.5)(-1.5)+(0.5)(1.5)+(1.5)(0.5)}{4 \sigma_{R(X)} \sigma_{R(Y)}} \\
& =\frac{0.75+0.75+0.75+0.75}{4(1.25)} \\
& =\frac{3}{5} \\
& =0.6
\end{aligned}
$$




\section{Wilcoxon Signed Rank}

Wilcoxon Signed Rank is another statistical test based on the rank of a sequence. It was used by Li et al. [LZCF17] for timing covert channel detection. Given two sequences $X$ and $Y$ of length $N$, let $D$ be the sequence $\left\{Y_{i}-X_{i} \mid \forall i \leq N, X_{i} \neq Y_{i}\right\}$. Thus, $D$ is the difference between each element of $X$ and $Y$ with all zeros removed. Let $R$ be the rank of the absolute values of $D$ and $S$ its sign:

$$
S_{i}=\left\{\begin{array}{l}
1, X_{i}>Y_{i} \\
-1, Y_{i}>X_{i}
\end{array}\right.
$$

The value of the Wilcoxon Signed Rank test is given by:

$$
W(X, Y)=\sum_{i} S_{i} R_{i}
$$

For the data in Table 3.2 one would obtain the intermediate sequences:

$$
\begin{gathered}
D(X, Y)=\{2-8,7-6,9-17,12-14\} \\
=\{6,-1,8,2\} \\
R(X, Y)=\{3,1,4,2\} \\
S(X, Y)=\{1,-1,1,1\}
\end{gathered}
$$


And thus a Wilcoxon Signed Rank test value of:

$$
\begin{aligned}
W(X, Y) & =\sum_{i} S_{i} R_{i} \\
& =(1)(3)+(-1)(1)+(1)(4)+(1)(2) \\
& =3-1+4+2 \\
& =8
\end{aligned}
$$

\section{Regularity}

Regularity is one of the rare metrics developed specifically for covert channels. It was proposed by Cabuk et al. [CBS04] for detecting covert channels using the timing of IP packets. It measures the changes in the standard deviation of a sequence. Given a sequence $X$ of length $N$ split it into non-overlapping windows of size $W$ (the final window can be truncated) and compute the standard deviation for each such windows. This results in the sequence shown in Equation 3.23, where $\sigma$ is a function returning

the standard deviation of the sequence it is given and $N_{W}=\left\lceil\frac{N}{W}\right\rceil$ is the number of windows:

$$
\left.S=\left\{\sigma\left\{X_{1} \ldots X_{W}\right\}, \sigma\left\{X_{W+1} \ldots X_{2 W}\right\} \ldots \sigma\left\{X_{i W+1} \ldots X_{(i+1) W}\right\} \ldots \sigma\left\{X_{N_{W}} \ldots X_{N}\right)\right\}\right\}
$$


With this, let $D$ be the percent difference between every unique pair of elements in $S$, that is:

$$
D=\frac{\left|S_{i}-S_{j}\right|}{S_{j}}, \forall i, j \leq N_{W}, i<j
$$

The regularity is the standard deviation of $D$.

For example, given the sequence $X=1,1,2,3,5,8,13,21,34$ of length 9 , one can split into 3 windows of length $3:\{\{1,1,2\},\{3,5,8\},\{13,21,34\}\}$ Computing the standard deviation of each windows yields:

$$
\begin{aligned}
S & =\{\sigma\{1,1,2\}, \sigma\{3,5,8\}, \sigma\{13,21,34\}\} \\
& =\{0.577,1.528,10.599\}
\end{aligned}
$$

And

$$
\begin{aligned}
D & =\frac{\left|S_{i}-S_{j}\right|}{S_{j}}, \forall i, j \leq N_{W}, i<j \\
& =\left\{\frac{\left|S_{1}-S_{2}\right|}{S_{2}}, \frac{\left|S_{1}-S_{3}\right|}{S_{3}} \frac{\left|S_{2}-S_{3}\right|}{S_{3}}\right\} \\
& =\left\{\frac{|0.577-1.528|}{1.528}, \frac{|0.528-10.599|}{10.599} \frac{|1.528-10.599|}{10.599}\right\} \\
& =\{0.622,0.950,0.856\}
\end{aligned}
$$

Finally, computing the standard deviation of $D$ gives a regularity of 0.169 . 


\section{Chapter 4}

\section{Experiment}

Having gathered an inventory of statistical tests that have the potential to be useful for detecting storage covert channels, the next step is to compare them. In so doing, we will determine which statistical tests are not suited to storage covert channel detection and, most importantly, which ones would make up the best covert channel detection system.

This chapter describes the experiment that was used to evaluate the ability of statistical tests to detect storage covert channels. Section 4.1 lays out the tests used in the experiment. Section 4.2 describes the process used to perform the experiment and Section 4.3 present the results. Finally, Section 4.4 discusses the results and draws conclusions. 
Table 4.1: Tests Used in the Experiment

\begin{tabular}{lll}
\hline Full Name & Abbreviation & Classification \\
\hline LZMA Compression & LZMA & Complexity \\
LZ77 Compression & LZ77 & Complexity \\
LZ78 Compression & LZ78 & Complexity \\
Lempel-Ziv Complexity & LZC & Complexity \\
First-Order Entropy & FOE & Complexity \\
Corrected Conditional Entropy & CCE & Complexity \\
Repetition & REP & Complexity \\
Autocovariance & COV & Distributional \\
Kolmogorov-Smirnov Test & KS & Distributional \\
Wilcoxon Signed Rank & WCX & Distributional \\
Spearman Correlation & SPR & Distributional \\
Regularity & REG & Distributional \\
\hline
\end{tabular}

\subsection{Tests Used in the Experiment}

The tests that are the subject of the experimentation presented in this chapter are catalogued in Table 4.1.

\subsection{Setup and Approach}

In this section, we describe our experimental setup and approach.

\subsubsection{Building the Dataset}

To build the dataset for our experiments, we needed samples of network traffic with and without information covertly embedded into them. Samples of real normal network traffic were acquired from the Malware Capture Facility Project at the Czech 
Technical University in Prague [Gar17]. As its name suggests, the project is aimed at analyzing malware, but they also provide normal traffic captures, which are what was used. The dataset contains 9,279, 501 packets of which 9,275,031 use IPv4 and were captured on 12 different occasions.

To generate network traffic with covert information, we chose to build a storagebased covert channel by embedding bits into the Identification (ID) field of the IP header. The purpose of this field is to identify packets that have been fragmented when the fragments are to be reconstructed. But today, networks tend to have large enough maximum transmissible units that IP packets do not need to be fragmented and these packets are usually sent with IP Flags set to "Don't fragment." Because of this, the ID field is rarely payed any attention, making it a good place to inject covert information. Furthermore, the field is included in every IP packet and is not expected to follow any particular pattern (unlike TCP Sequence numbers), so new covert information can be included in every packet sent. The field is 16 bits long, so a covert channel could transmit up to two bytes per packet.

For our experiments, a covert message had to be selected. The covert message ought to be something that any test would view as similar to real human communication. Text from a novel is appropriate for this purpose, and we chose to use the first few paragraphs of Pride and Prejudice by Jane Austen. The specific text is reproduced in Appendix A.

To create test data, the IP ID fields were extracted from each of the IP headers. Bits from the message were embedded into the these IP ID fields by replacing the 
least significant bits of the field with bits from the message ${ }^{1}$. The number of bits replaced was varied between 1 and 16, i.e., between changing just one bit of the field, and replacing them completely. Henceforth, the sequence of IP ID values extracted from the network before the message is embedded will be referred to as the carrier; once these carriers have message bits embedded into them they will be known as traces. Each 16-bit IP ID value (with or without message bits embedded) that form the traces/carrier will be known as an element. Note that to create a trace, the least significant bits of each element of the carrier were replaced completely (i.e., AND-ed with zeroes and then OR-ed with the message bits). Using an exclusive-or operation to embed information does not work in this case because the message recipient does not know the value of the field in the carrier and so cannot recover the message if it is embedded via exclusive-or. For example, to embed the first two bits of the word "The" into the IP ID value 0x154A8FE0E, we set the two least significant bits to zero to get $0 \times 154 \mathrm{~A} 8 \mathrm{FE} 0 \mathrm{C}$ and $\mathrm{OR}$ that with '01' - the first two bits of $0 \times 54$, the value of ' $\mathrm{T}$ ' in ASCII - to get $0 \mathrm{x} 154 \mathrm{~A} 8 \mathrm{FE} 0 \mathrm{D}$. The result of the entire process is a set of 16 different traces, one for each number of bits. To ensure that comparisons between them are valid, all 16 traces are of the same length and they all contain the entire message. This requires that all traces except the 1-bit trace contain IP ID fields with no message content.

\footnotetext{
${ }^{1} \mathrm{~A}$ similar process can be adopted for other header fields of network packets.
} 


\subsubsection{Conducting the Experiments}

The code for generating the datasets and conducting our experiments is available at: gitlab.com/CyberSEA-Public/CCStatTests. The experiments are done in iterations. On each iteration the IP ID field is extracted from a certain number of different packets creating a carrier. The message is then embedded, creating 16 traces (as described in Section 4.2.1) and the tests are applied to each trace. The result is one value per test per trace. The tests are also applied to the carrier independently of the tests on the traces.

In the next iteration each of these tests is repeated. In total, 1000 iterations are performed and we calculate the mean and standard deviation for each trace/carrier. This whole process is repeated 3 times so as to vary the size of the message. Tests with 256, 16 and 1 byte messages are performed. Each message bit of each iteration requires its own unique IP ID value, thus $256 \times 8 \times 1000=2,048,000$ packets are used. The underlying packets used are randomized across iterations; what is meant by this is that within a given iteration the packets used for sequential message bits are sequential in the original dataset, but the packets used in the subsequent iteration start at another random place in the dataset. This allows the predictor to exploit patterns expressed by sequential IP ID values while still preventing the results from being skewed excessively by the idiosyncrasies of any particular capture period.

In addition to the repeated iterations and different message sizes, the process was repeated with the message encrypted in order to investigate the effect of encryption on the tests' performance. The Rijndael cipher of the Advanced Encryption Standard (AES) is the current state of the art, so this is what we used. However, AES uses a 
block cipher with 16-byte blocks such that the smallest message that can be encrypted is 16 bytes long ${ }^{2}$. Consequently, a stream cipher that can encrypt a single byte was used for the 1-byte messages, namely the Salsa20 cipher. This limitation is the reason for using 16 bytes as the second smallest message size in our experiments.

\subsection{Results}

In this section we report the results of our experiments following the approach described in Section 4.2.

\subsubsection{Results for Tests Used in Isolation}

The results of each experiment are presented here as a series of tables. First Tables 4.2-4.4 present the effect size for every trace for each message size for each test in our experiments. More specifically, they show the difference between the mean of the test's output on the traces and the mean output on the carrier. This difference is presented in units of the pooled standard deviation of the carrier and trace outputs (i.e., the square root of the mean of the two variances). This is known as the effect size and it measures how clearly the test can distinguish each trace from the carrier (see Section 3.1).

The first thing to note is that complexity tests perform much better than distributional tests. No distributional test ever produced an effect size greater than 0.00189

\footnotetext{
${ }^{2}$ Padding the message would complicate interpretation of the results.
} 
Table 4.2: Effect Sizes for 256-byte messages in an IP ID covert channel

\begin{tabular}{lllllll}
\hline & 1-bit Trace & \multicolumn{3}{c}{ Minimum } & \multicolumn{3}{c}{ Min. Index } \\
\hline Encrypted? & Y & $\mathrm{N}$ & $\mathrm{Y}$ & $\mathrm{N}$ & $\mathrm{Y}$ & $\mathrm{N}$ \\
\hline LZMA & 2.95 & 3 & 2.95 & 3 & 1 & 1 \\
LZ77 & 6.3 & 4.62 & 3.59 & 3.55 & 3 & 2 \\
LZ78 & 3.56 & 3.25 & 3.56 & 3.25 & 1 & 1 \\
LZC & 5.04 & 4.28 & 5.04 & 4.28 & 1 & 1 \\
FOE & 3.37 & 3.13 & 3.37 & 3.13 & 1 & 1 \\
CCE & 0.445 & 0.449 & 0.445 & 0.449 & 1 & 1 \\
REP & 4.58 & 3.94 & 4.58 & 3.94 & 1 & 1 \\
COV & $3.08 \mathrm{e}-08$ & $9.12 \mathrm{e}-07$ & $3.08 \mathrm{e}-08$ & $4.23 \mathrm{e}-07$ & 1 & 3 \\
KS & 0.00189 & 0.00184 & 0.00189 & 0.00184 & 1 & 1 \\
WCX & $1.26 \mathrm{e}-05$ & $2.53 \mathrm{e}-05$ & $1.26 \mathrm{e}-05$ & $2.53 \mathrm{e}-05$ & 1 & 1 \\
SPR & $3.46 \mathrm{e}-06$ & $1.31 \mathrm{e}-05$ & $3.46 \mathrm{e}-06$ & $1.31 \mathrm{e}-05$ & 1 & 1 \\
REG & $8.77 \mathrm{e}-07$ & $3.82 \mathrm{e}-06$ & $8.77 \mathrm{e}-07$ & $3.82 \mathrm{e}-06$ & 1 & 1 \\
\hline
\end{tabular}

Table 4.3: Effect Sizes for 16-byte messages in an IP ID covert channel

\begin{tabular}{lllllll}
\hline & 1-bit Trace & & Minimum & & Min. Index \\
\hline Encrypted? & Y & N & Y & N & Y & N \\
\hline LZMA & 4.87 & 4.76 & 4.87 & 4.76 & 1 & 1 \\
LZ77 & 3.24 & 3.15 & 2.58 & 3.15 & 2 & 1 \\
LZ78 & 6.9 & 5.98 & 6.9 & 5.98 & 1 & 1 \\
LZC & 4.25 & 4.05 & 4.25 & 4.05 & 1 & 1 \\
FOE & 6.24 & 5.55 & 6.24 & 5.55 & 1 & 1 \\
CCE & 2.64 & 2.63 & 2.64 & 2.63 & 1 & 1 \\
REP & 8.25 & 6.7 & 8.25 & 6.7 & 1 & 1 \\
COV & $6.88 \mathrm{e}-07$ & $1.19 \mathrm{e}-06$ & $6.88 \mathrm{e}-07$ & $1.19 \mathrm{e}-06$ & 1 & 1 \\
KS & 0.000624 & 0.00125 & 0.000624 & 0.00125 & 1 & 1 \\
WCX & $3.95 \mathrm{e}-06$ & $8.82 \mathrm{e}-05$ & $3.95 \mathrm{e}-06$ & $8.82 \mathrm{e}-05$ & 1 & 1 \\
SPR & 0.00109 & 0.000826 & 0.00109 & 0.000826 & 1 & 1 \\
REG & $\perp$ & $\perp$ & $\perp$ & $\perp$ & $\perp$ & $\perp$ \\
\hline
\end{tabular}


Table 4.4: Effect Sizes for 1-byte messages in an IP ID covert channel

\begin{tabular}{lllllll}
\hline & 1-bit Trace & & Minimum & & Min. Index \\
\hline Encrypted? & $\mathrm{Y}$ & $\mathrm{N}$ & $\mathrm{Y}$ & $\mathrm{N}$ & $\mathrm{Y}$ & $\mathrm{N}$ \\
\hline LZMA & $\perp$ & $\perp$ & $\perp$ & $\perp$ & $\perp$ & $\perp$ \\
LZ77 & $\perp$ & $\perp$ & $\perp$ & $\perp$ & $\perp$ & $\perp$ \\
LZ78 & 0.135 & 0.165 & 0.135 & 0.165 & 1 & 1 \\
LZC & 0.0164 & 0.0106 & 0.0164 & 0.0106 & 1 & 1 \\
FOE & 0.139 & 0.169 & 0.139 & 0.169 & 1 & 1 \\
CCE & 0.139 & 0.171 & 0.139 & 0.171 & 1 & 1 \\
REP & 0.139 & 0.169 & 0.139 & 0.169 & 1 & 1 \\
COV & $3.32 \mathrm{e}-06$ & $3.45 \mathrm{e}-07$ & $6.66 \mathrm{e}-07$ & $3.45 \mathrm{e}-07$ & 2 & 1 \\
KS & 0.00157 & 0 & 0 & 0 & 2 & 1 \\
WCX & 0 & 0 & 0 & 0 & 1 & 1 \\
SPR & 0.000775 & 0.000996 & $9.73 \mathrm{e}-05$ & $9.73 \mathrm{e}-05$ & 7 & 7 \\
REG & $\perp$ & $\perp$ & $\perp$ & $\perp$ & $\perp$ & $\perp$ \\
\hline
\end{tabular}

(see Table 4.2) and, excluding the 1-byte messages (Table 4.4), all the complexity tests results are at least 0.445 (Table 4.2) - a difference of more than 2 orders of magnitude. If we go further and exclude Corrected Conditional Entropy on 256-byte messages the weakest results for a complexity test is much larger still at 2.58 (Table 4.3). As for the 1-byte messages, several test results were undefined (denoted by $\perp$ ). This means that the difference in the mean and joint standard deviations were both zero, i.e., the test result was the same for every iteration for both the carrier and at least one trace. In context, this means the test fails to differentiate the traces from the carrier, so $\perp$ is roughly equivalent to 0 . That this is the result for many of the complexity tests means that a 1-byte message is not sufficient for them to usefully detect covert channels; even the tests that avoid this issue do not produce large effects.

Moreover, encryption made little difference and, counterintuitively, the tests mostly did better when the message was encrypted. This improvement is nonetheless quite small; LZ77 was affected the most and even it has a large effect everywhere (except 


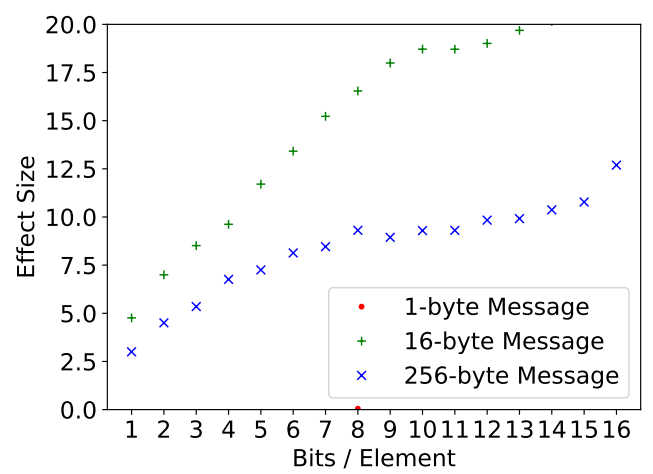

Figure 4.1: LZMA Effect Sizes for IP ID

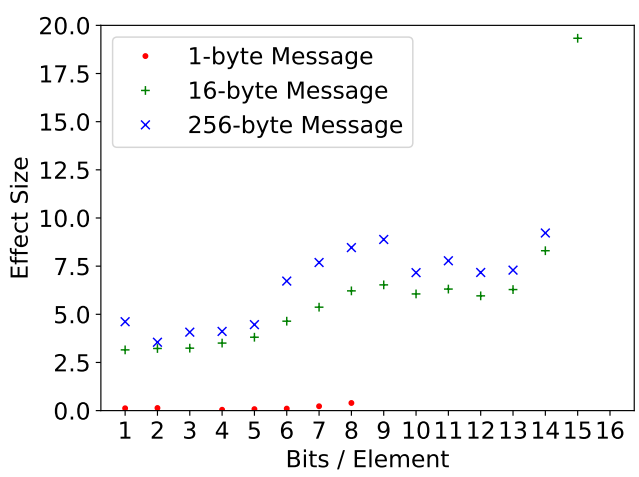

Figure 4.2: LZ77 Effect Sizes for IP ID

on 1-byte messages) regardless of encryption.

Next, it is interesting to note that 1-bit traces are generally the least different from the carrier. The only exceptions to this, besides the distributional tests and the 1-byte message table where the tests are not effective, are some of the LZ77 results. This trend is more thoroughly visualized in Figures 4.1 to 4.12 in which the noisy trend of increasing effect size with more message bits per element is evident. Note that the graphs lack datapoints for 1-byte messages with more than 8 bits per element because the message as a whole contains only 8 bits.

One of the most interesting things about the result is that Repetition outperformed both First-Order Entropy and Corrected Conditional Entropy. This is odd because these both rely heavily on counting unique elements. The main difference is that entropy-based tests do so in a way that properly reflects the information contained in the traces and takes into account elements that are repeated more than once, whereas Repetition is ad hoc and should not be expected to be very meaningful. Nevertheless, 


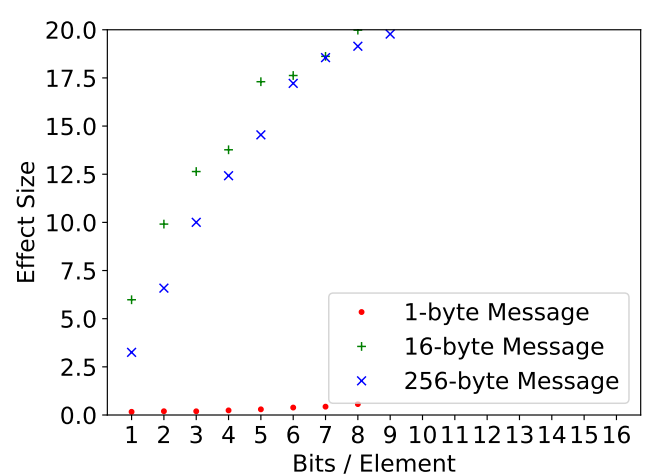

Figure 4.3: LZ78 Effect Sizes for IP ID

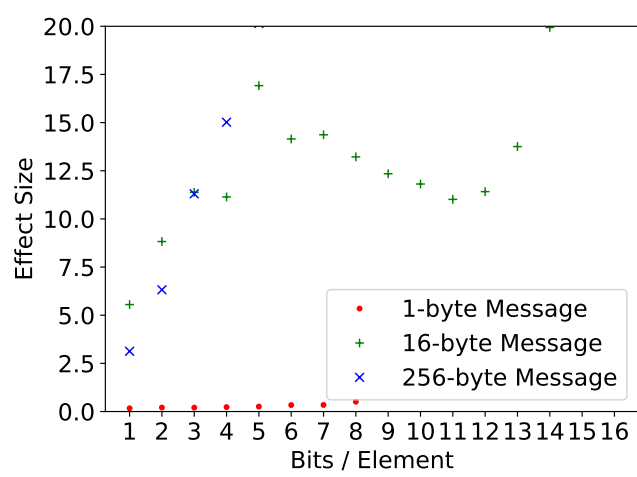

Figure 4.5: FOE Effect Sizes for IP ID

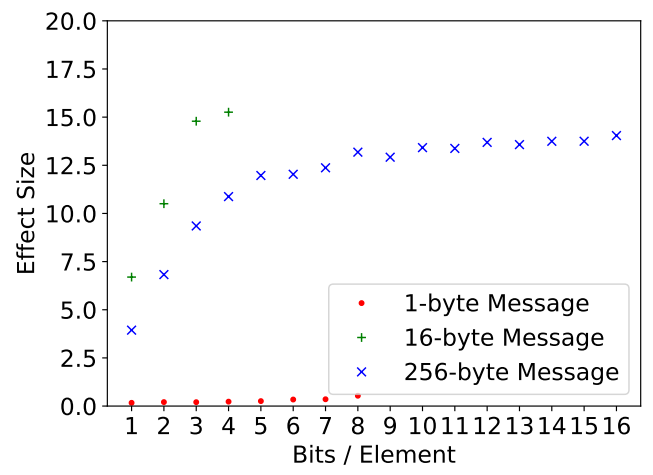

Figure 4.7: REP Effect Sizes for IP ID

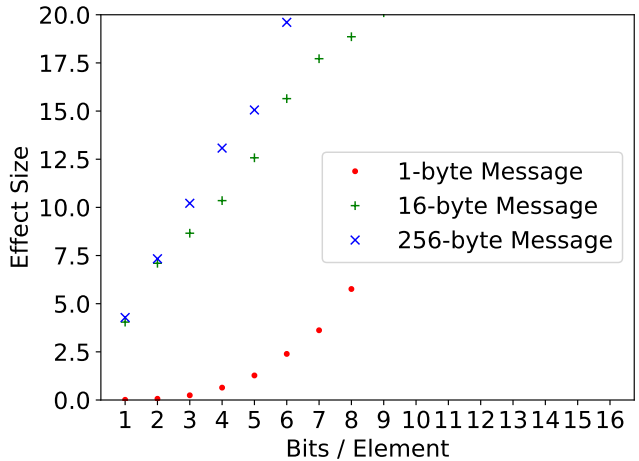

Figure 4.4: LZC Effect Sizes for IP ID

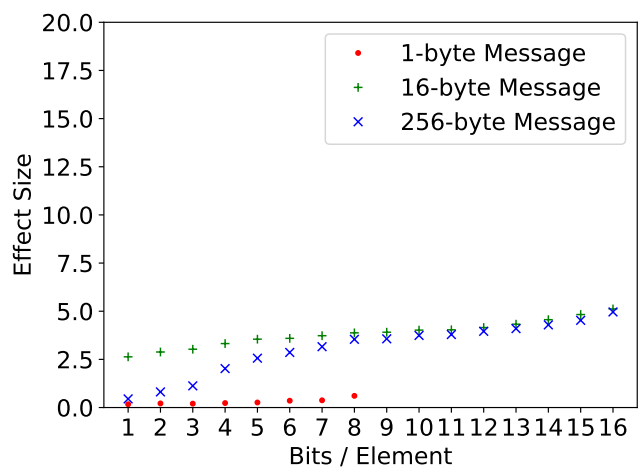

Figure 4.6: CCE Effect Sizes for IP ID

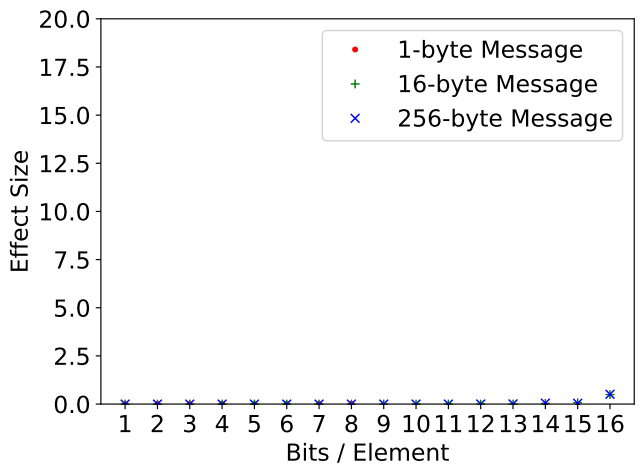

Figure 4.8: COV Effect Sizes for IP ID 


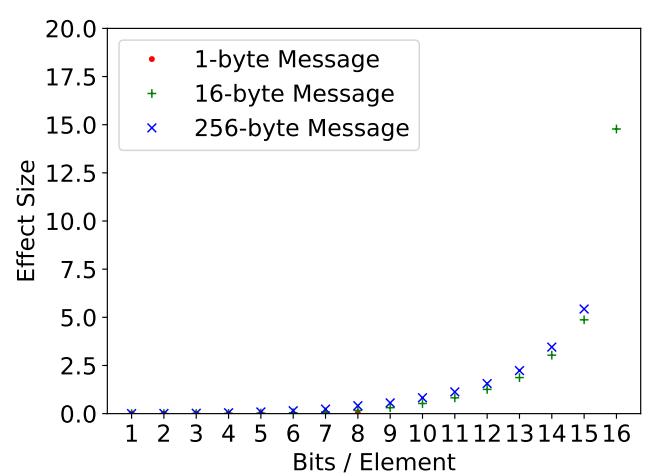

Figure 4.9: KS Effect Sizes for IP ID

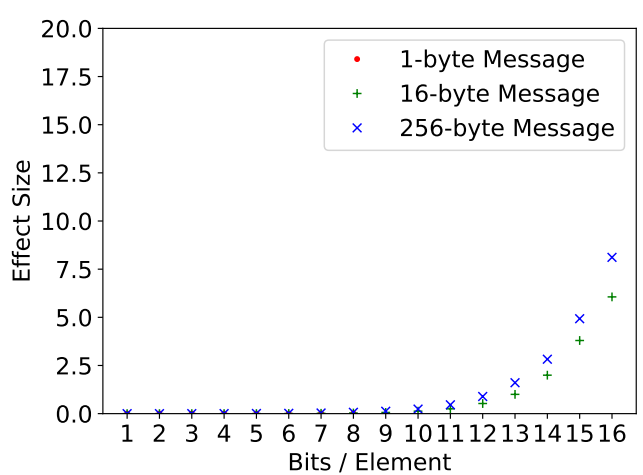

Figure 4.10: WCX Effect Sizes for IP ID

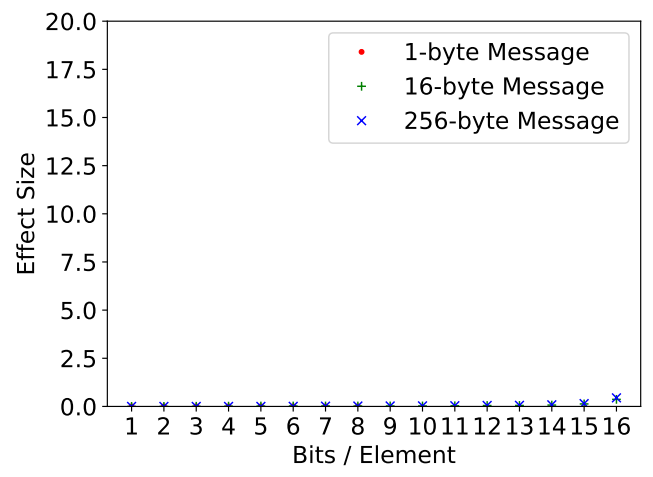

Figure 4.11: SPR Effect Sizes for IP ID

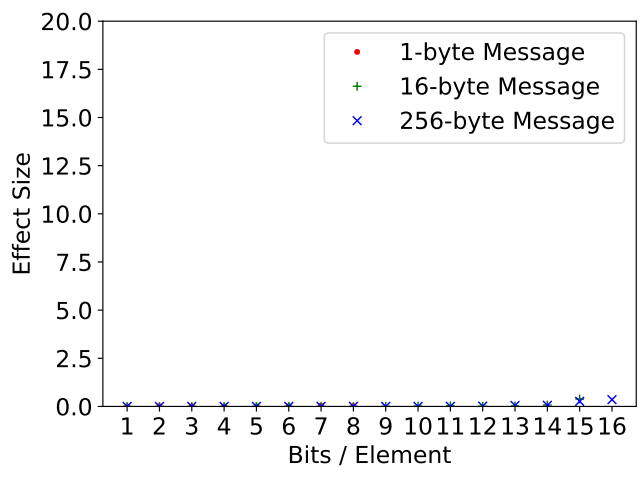

Figure 4.12: REG Effect Sizes for IP ID 
Repetition performs better and is certainly more efficient than either of the entropybased tests: the computations required for Repetition are a strict subset of those required for First-Order Entropy which are themselves a strict subset of those for Corrected Conditional Entropy.

As for the compression-based tests and Lempel-Ziv Complexity, all of them perform similarly and on par with Repetition on 256-byte messages. On 16-byte messages, LZ77 is notably worse and LZ78 is notably better, but all are outperformed by Repetition. This analysis tentatively pinpoints Repetition as the best test overall with LZ78 close behind, but there is no clear winner.

\subsubsection{Results for Tests Used in Combination}

Tests need not be used in isolation of each other and it is of interest to determine how they perform together. To do this, we show the correlation between the results of the tests across iterations in Tables 4.5 and 4.6. Because, in the previous section, the 1-bit traces were the hardest to detect, we restrict our analysis here to those and because encryption had no significant impact, we no longer keep it in our consideration. We also do not continue to analyze distributional tests and 1-byte messages, having concluded that a storage-based covert channel detector based on these ideas is not effective. To be clear, what is being compared is the correlation across all iterations in the difference between the output of the tests for 1-bit traces and the carrier.

Surprisingly, the compression-based tests are not clearly more correlated with each 
Table 4.5: Correlation for 1-bit traces of 256-byte messages in an IP ID covert channel

\begin{tabular}{llllllll}
\hline & LZMA & LZ77 & LZ78 & LZC & FOE & CCE & REP \\
\hline LZMA & 1 & 0.25 & 0.23 & 0.19 & 0.18 & 0.18 & 0.16 \\
LZ77 & 0.25 & 1 & 0.66 & 0.76 & 0.68 & 0.13 & 0.65 \\
LZ78 & 0.23 & 0.66 & 1 & 0.87 & 0.94 & 0.15 & 0.94 \\
LZC & 0.19 & 0.76 & 0.87 & 1 & 0.92 & 0.17 & 0.94 \\
FOE & 0.18 & 0.68 & 0.94 & 0.92 & 1 & 0.14 & 0.99 \\
CCE & 0.18 & 0.13 & 0.15 & 0.17 & 0.14 & 1 & 0.14 \\
REP & 0.16 & 0.65 & 0.94 & 0.94 & 0.99 & 0.14 & 1 \\
\hline
\end{tabular}

Table 4.6: Correlation for 1-bit traces of 16-byte messages in an IP ID covert channel

\begin{tabular}{llllllll}
\hline & LZMA & LZ77 & LZ78 & LZC & FOE & CCE & REP \\
\hline LZMA & 1 & 0.65 & 0.59 & 0.71 & 0.59 & 0.19 & 0.58 \\
LZ77 & 0.65 & 1 & 0.50 & 0.71 & 0.51 & 0.064 & 0.51 \\
LZ78 & 0.59 & 0.50 & 1 & 0.69 & 0.97 & 0.20 & 0.97 \\
LZC & 0.71 & 0.71 & 0.69 & 1 & 0.70 & 0.11 & 0.70 \\
FOE & 0.59 & 0.51 & 0.97 & 0.70 & 1 & 0.18 & 1 \\
CCE & 0.19 & 0.064 & 0.20 & 0.11 & 0.18 & 1 & 0.18 \\
REP & 0.58 & 0.51 & 0.97 & 0.70 & 1 & 0.18 & 1 \\
\hline
\end{tabular}

other than with the entropy-based ones. The general trend is that tests are moderately correlated with each other. There are, however, some definite outliers. First of all, First-Order Entropy and Repetition are very strongly correlated; this diminishes First-Order Entropy as a contender because Repetition outperforms it and gives very similar results from one iteration to the next. Secondly, Corrected Conditional Entropy and LZMA Compression have relatively weak correlation with the other tests. The weakness of these correlations suggest that two tests could be combined to make a more effective detector. In fact, except for First-Order Entropy and Repetition, any number of the tests could be combined because their correlations are not close to perfect. 


\subsubsection{Logistic Regression Detector}

In this section, we evaluate the performance of covert channel detectors that use the tests evaluated herein. The algorithm used to create the detector is a simple logistic regression classifier. This technique takes in negative and positive examples and returns a model that estimates the probability that a certain sample is positive based on its Euclidean distance from a line. The number of tests determines the dimensionality of the space in which the linear classifier exists. In other words, the features of the logistic regression model are the outputs of one or more of the tests. Performing feature selection is not required because a maximum of seven dimensions is easily manageable with regards to computational demands.

The positive examples are the test results for the 1-bit traces and the negative examples are the test results for the carrier. The examples are split into a training set and a test set so that the model can be evaluated on examples it has not yet seen. $70 \%$ of examples are in the training set leaving $30 \%$ for the test set. There are 2000 examples in total; two - a negative and a positive - for each of the 1000 iterations. This yields a training set of 1400 examples and a test set of 600 examples; both are balanced (i.e., they contain roughly the same number of positive and negative examples). All this is repeated for four message sizes: 256, 64, 16, and 4 bytes. The creation of the classifier was repeated 1000 times, randomizing the examples that were included in the test set in order to average out any effect of how the dataset is split; both the mean and the standard deviation are reported.

The accuracy of the classifier for an IP ID covert channel is presented in Table 4.7. Each column represents the accuracy for a different message size while each row except 
Table 4.7: Detector accuracy for IP ID covert channel for various message sizes

\begin{tabular}{lllll}
\hline Message Size & 256 bytes & 64 bytes & 16 bytes & 4 bytes \\
\hline LZMA & $0.950 \pm 0.0086$ & $0.966 \pm 0.0066$ & $0.985 \pm 0.0040$ & $0.526 \pm 0.038$ \\
LZ77 & $0.998 \pm 0.0013$ & $0.995 \pm 0.0023$ & $0.973 \pm 0.0054$ & $0.908 \pm 0.0099$ \\
LZ78 & $0.976 \pm 0.0056$ & $0.992 \pm 0.003$ & $0.997 \pm 0.0021$ & $0.945 \pm 0.0093$ \\
LZC & $0.966 \pm 0.11$ & $0.995 \pm 0.0024$ & $0.969 \pm 0.0061$ & $0.74 \pm 0.015$ \\
FOE & $0.982 \pm 0.0046$ & $0.995 \pm 0.0027$ & $0.997 \pm 0.0017$ & $0.984 \pm 0.012$ \\
CCE & $0.996 \pm 0.002$ & $0.998 \pm 0.0015$ & $0.930 \pm 0.0086$ & $0.982 \pm 0.0046$ \\
REP & $0.984 \pm 0.0043$ & $0.995 \pm 0.0024$ & $0.999 \pm 0.0013$ & $0.953 \pm 0.021$ \\
All & $0.992 \pm 0.0038$ & $0.995 \pm 0.0025$ & $0.991 \pm 0.0038$ & $0.982 \pm 0.0045$ \\
\hline
\end{tabular}

for the last represents the accuracy of a detector that uses only one of the complexity tests. The bottom row (All) shows the accuracy of a detector using all the tests together. The first thing to note about the results is that a detector using just one of these tests works very well: most of the detectors are more than $90 \%$ accurate even for messages as small as 4 bytes and some are more than $95 \%$ accurate. For messages of 16 bytes or more, the accuracy climbs to over $99 \%$ in most cases. The second thing to note is that the detector using all the tests does not outperform those that use just one test. Given this, it seems that combining more than one of the tests to create a more effective detector just leads to unnecessary complexity in the detector design.

The impact of combining tests for a logistic regression detector can be visualized by plotting the probability estimates of a 2-test - and thus 2-dimensional - classifier. This was done for Repetition and Lempel-Ziv Complexity on 256-byte, 64-byte, 16byte and 4-byte messages in Figures 4.13 to 4.16 respectively. Each figure uses a background colour transition from red to blue to show how the classifier assigns a probability to each point on the plane. The positive examples are depicted in blue and the negative examples in red. The set of examples is normalized to zero mean and unit 


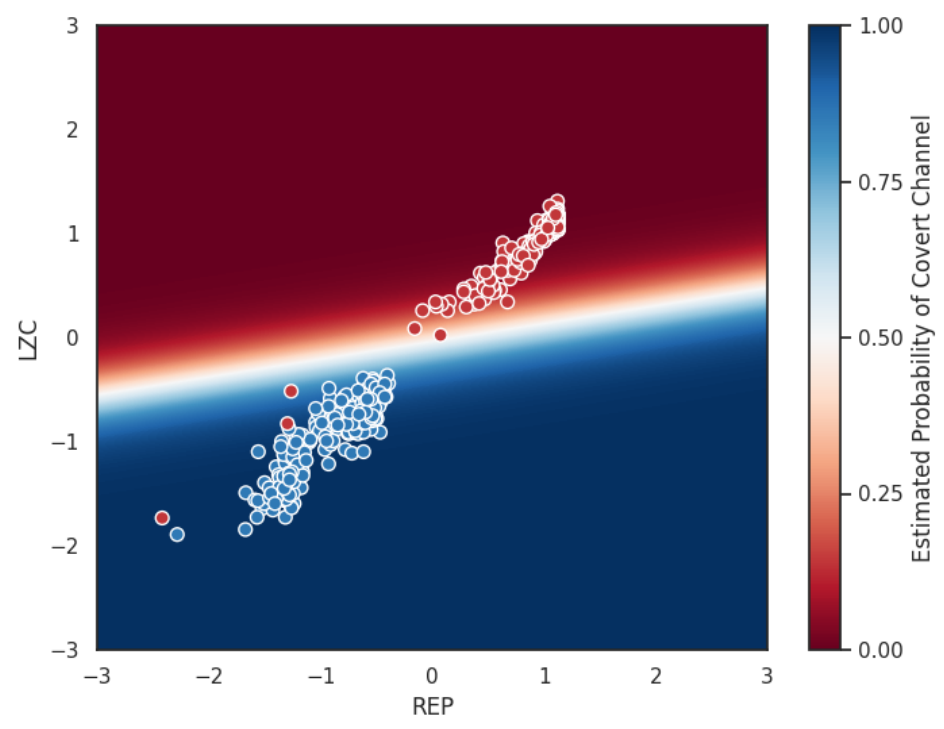

Figure 4.13: 256-byte Message REP-LZC Detector Probability Estimates

standard deviation, guaranteeing that almost all points will be present in a 6 by 6 plot centered on the origin. Figure 4.16 is interesting in that the boundaries between zones of different colours are almost vertical. This indicates that the estimate for 4 -byte messages is almost entirely driven by the Repetition test values, plotted on the $\mathrm{X}$-axis. Conversely, the boundaries in Figure 4.13 are nearly horizontal because Lempel-Ziv Complexity dominates. Figures 4.14 and 4.15 have more angled boundaries indicating that both tests are used. However, it is the 4-byte case in Figure 4.16 that is the most challenging to predict (without being futile like the 1-byte case) and a practical detector should ideally have to process as few packets as possible before making a prediction, so performance there is most important.

What one can take away from this section, is that logistic regression is up to the task of detecting the use an IP ID covert channel using the outputs of complexity tests. 


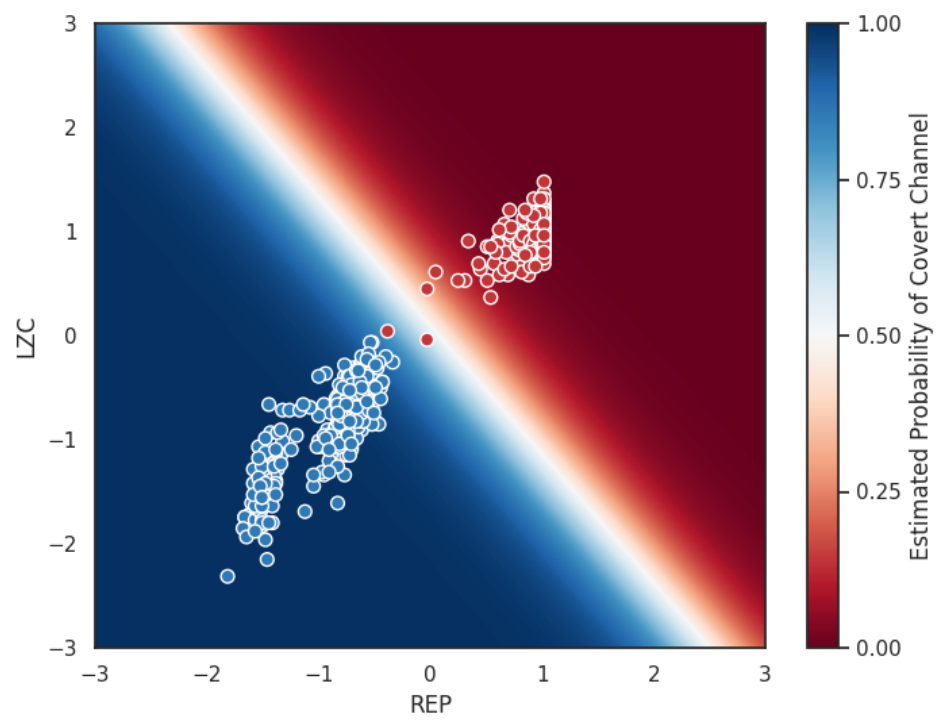

Figure 4.14: 64-byte Message REP-LZC Detector Probability Estimates

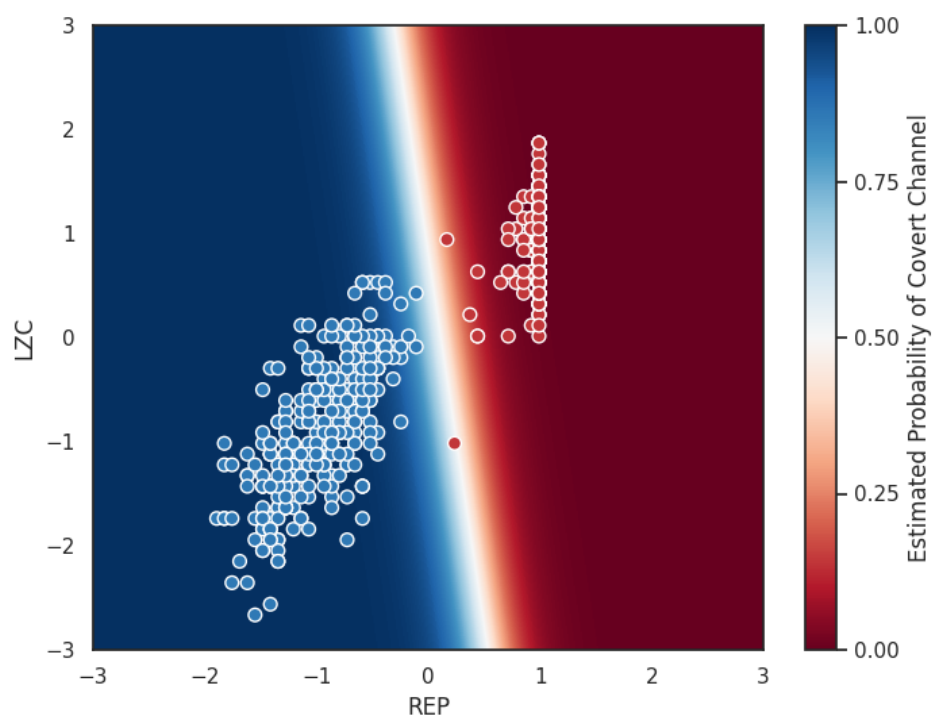

Figure 4.15: 16-byte Message REP-LZC Detector Probability Estimates 


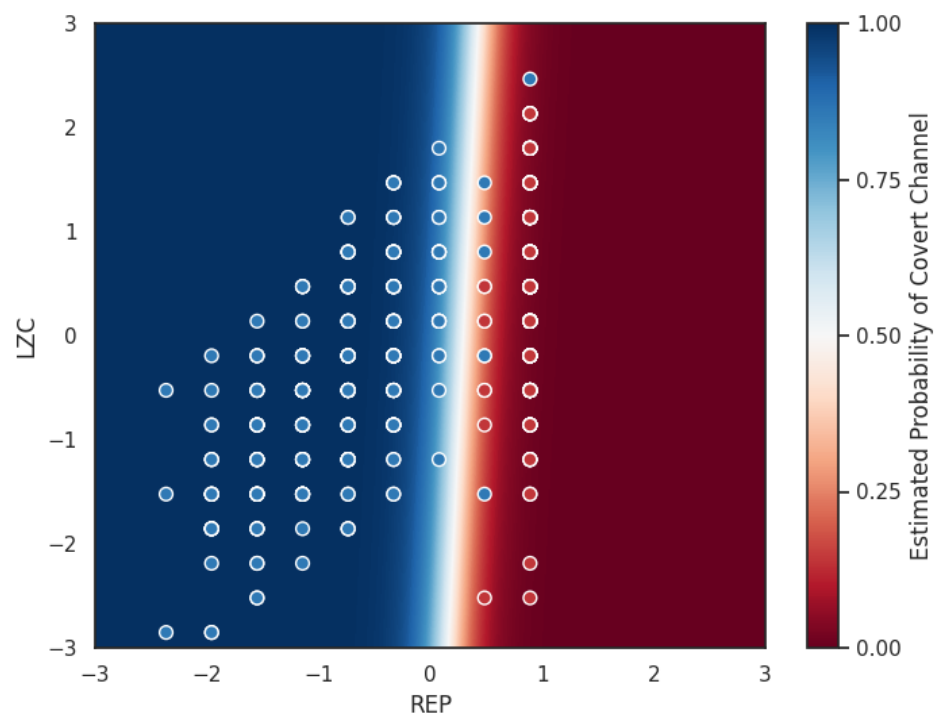

Figure 4.16: 4-byte Message REP-LZC Detector Probability Estimates

This is not to say that a more sophisticated classifier could not perform even better, but it would struggle to do much better as that would involve reducing the number of misclassified samples to a very low number. Moreover, logistic regression has the advantage that the classification boundary it produces is a simple linear function. This is particularly important given the goal of building a real-time detector in hardware where implementing a classier that performs a complex operation to predict each sample would be challenging.

\subsubsection{Repeating the Experiment for TCP ISN}

Having performed this experiment on storage-based covert channels using the IP ID field it seemed natural to investigate covert channels using other fields such as TCP Initial Sequence Numbers (ISN). This was done to underwhelming results. Effect 
Table 4.8: Detector accuracy for TCP ISN covert channel for various message sizes

\begin{tabular}{lllll}
\hline Message Bytes & 256 bytes & 64 bytes & 16 bytes & 4 bytes \\
\hline LZMA & $0.415 \pm 0.061$ & $0.412 \pm 0.062$ & $0.408 \pm 0.064$ & $0.407 \pm 0.069$ \\
LZ77 & $0.415 \pm 0.06$ & $0.412 \pm 0.063$ & $0.410 \pm 0.063$ & $0.403 \pm 0.064$ \\
LZ78 & $0.412 \pm 0.063$ & $0.408 \pm 0.064$ & $0.409 \pm 0.064$ & $0.412 \pm 0.063$ \\
LZC & $0.412 \pm 0.063$ & $0.411 \pm 0.065$ & $0.404 \pm 0.075$ & $0.392 \pm 0.087$ \\
FOE & $0.414 \pm 0.061$ & $0.411 \pm 0.062$ & $0.410 \pm 0.065$ & $0.413 \pm 0.066$ \\
CCE & $0.410 \pm 0.065$ & $0.400 \pm 0.078$ & $0.430 \pm 0.098$ & $0.417 \pm 0.075$ \\
REP & $0.414 \pm 0.061$ & $0.408 \pm 0.068$ & $0.412 \pm 0.063$ & $0.409 \pm 0.064$ \\
All & $0.415 \pm 0.059$ & $0.384 \pm 0.071$ & $0.403 \pm 0.097$ & $0.378 \pm 0.089$ \\
\hline
\end{tabular}

Table 4.9: Effect Sizes for 256-byte messages in a TCP ISN covert channel

\begin{tabular}{lllllll}
\hline & 1-bit Trace & & Minimum & \multicolumn{3}{c}{ Min. Index } \\
\hline Encrypted? & $\mathrm{Y}$ & $\mathrm{N}$ & $\mathrm{Y}$ & $\mathrm{N}$ & $\mathrm{Y}$ & $\mathrm{N}$ \\
\hline LZMA & 0.423 & 0.416 & 0.179 & 0.161 & 3 & 3 \\
LZ77 & 0.136 & 0.182 & 0.0294 & 0.0294 & 6 & 6 \\
LZ78 & 0.327 & 0.334 & 0.0922 & 0.132 & 2 & 2 \\
LZC & 0.262 & 0.216 & 0.0265 & 0.0089 & 2 & 2 \\
FOE & 0.844 & 0.841 & 0.0331 & 0.032 & 14 & 14 \\
CCE & 0.867 & 0.881 & 0.0803 & 0.0803 & 15 & 15 \\
REP & 0.765 & 0.763 & 0.00866 & 0.00743 & 14 & 14 \\
COV & $4.05 \mathrm{e}-11$ & $1.37 \mathrm{e}-10$ & $4.05 \mathrm{e}-11$ & $1.37 \mathrm{e}-10$ & 1 & 1 \\
KS & 0 & 0 & 0 & 0 & 1 & 1 \\
WCX & 0 & 0 & 0 & 0 & 1 & 1 \\
SPR & $4.55 \mathrm{e}-05$ & $5.31 \mathrm{e}-06$ & $6.35 \mathrm{e}-06$ & $5.31 \mathrm{e}-06$ & 10 & 1 \\
REG & $6.89 \mathrm{e}-10$ & $1.45 \mathrm{e}-10$ & $1.78 \mathrm{e}-10$ & $1.45 \mathrm{e}-10$ & 3 & 1 \\
\hline
\end{tabular}


Table 4.10: Effect Sizes for 16-byte messages in a TCP ISN covert channel

\begin{tabular}{lllllll}
\hline & 1-bit Trace & \multicolumn{3}{c}{ Minimum } & \multicolumn{3}{c}{ Min. Index } \\
\hline Encrypted? & $\mathrm{Y}$ & $\mathrm{N}$ & $\mathrm{Y}$ & $\mathrm{N}$ & $\mathrm{Y}$ & $\mathrm{N}$ \\
\hline LZMA & 0.105 & 0.146 & 0.0555 & 0.0555 & 5 & 5 \\
LZ77 & 0.126 & 0.127 & 0 & 0 & 7 & 7 \\
LZ78 & 0.102 & 0.153 & 0.0865 & 0.0728 & 3 & 3 \\
LZC & 0.0211 & 0.118 & 0.0211 & 0.118 & 1 & 1 \\
FOE & 0.312 & 0.366 & 0.00819 & 0.00819 & 17 & 17 \\
CCE & 0.201 & 0.201 & 0.00129 & 0.00129 & 11 & 11 \\
REP & 0.301 & 0.35 & 0.0142 & 0.0142 & 17 & 17 \\
COV & $7.11 \mathrm{e}-11$ & $1.82 \mathrm{e}-11$ & $5.76 \mathrm{e}-11$ & $1.82 \mathrm{e}-11$ & 3 & 1 \\
KS & 0 & 0 & 0 & 0 & 1 & 1 \\
WCX & 0 & 0 & 0 & 0 & 1 & 1 \\
SPR & 0.000329 & $8.02 \mathrm{e}-05$ & $1.32 \mathrm{e}-06$ & $1.32 \mathrm{e}-06$ & 11 & 11 \\
REG & $\perp$ & $\perp$ & $\perp$ & $\perp$ & $\perp$ & $\perp$ \\
\hline
\end{tabular}

Table 4.11: Effect Sizes for 1-byte messages in a TCP ISN covert channel

\begin{tabular}{lllllll}
\hline & 1-bit Trace & & Minimum & & Min. Index \\
\hline Encrypted? & $\mathrm{Y}$ & $\mathrm{N}$ & $\mathrm{Y}$ & $\mathrm{N}$ & $\mathrm{Y}$ & $\mathrm{N}$ \\
\hline LZMA & $\perp$ & $\perp$ & $\perp$ & $\perp$ & $\perp$ & $\perp$ \\
LZ77 & 0.0744 & 0.0506 & 0 & 0 & 7 & 7 \\
LZ78 & 0.0351 & 0.0351 & 0 & 0 & 4 & 4 \\
LZC & 0.133 & 0.0384 & 0.055 & 0.0384 & 3 & 1 \\
FOE & 0.0787 & 0.142 & 0 & 0 & 8 & 8 \\
CCE & 0.0624 & 0.0624 & 0 & 0 & 8 & 8 \\
REP & 0.0686 & 0.126 & 0 & 0 & 8 & 8 \\
COV & $1.03 \mathrm{e}-10$ & $3.23 \mathrm{e}-10$ & $5.69 \mathrm{e}-12$ & $2.26 \mathrm{e}-10$ & 6 & 3 \\
KS & 0 & 0 & 0 & 0 & 1 & 1 \\
WCX & 0 & 0 & 0 & 0 & 1 & 1 \\
SPR & 0.00905 & 0.00758 & 0 & 0 & 8 & 8 \\
REG & $\perp$ & $\perp$ & $\perp$ & $\perp$ & $\perp$ & $\perp$ \\
\hline
\end{tabular}




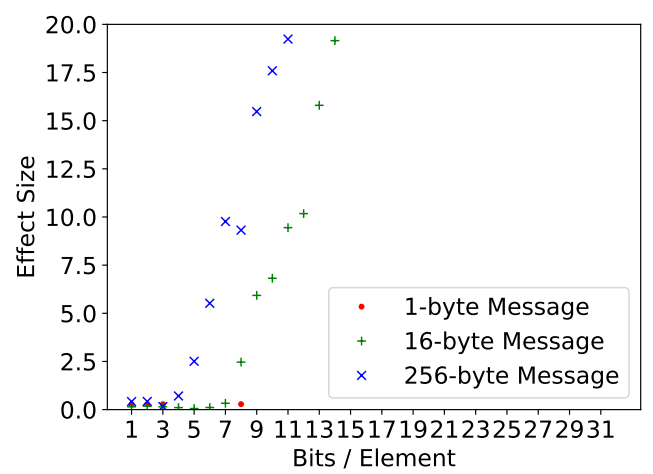

Figure 4.17: LZMA Effect Sizes for TCP ISN

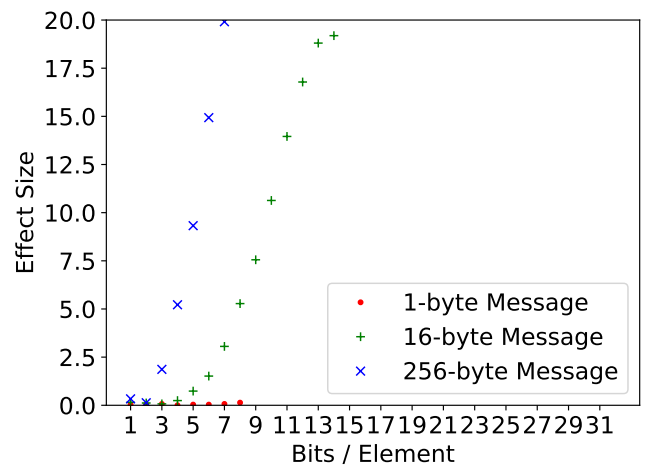

Figure 4.19: LZ78 Effect Sizes for TCP ISN

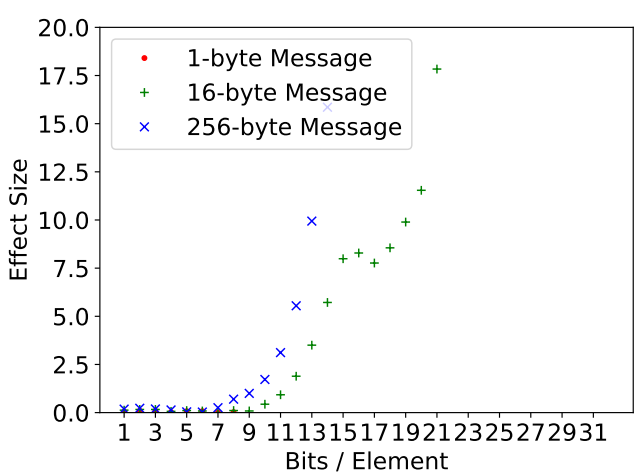

Figure 4.18: LZ77 Effect Sizes for TCP ISN

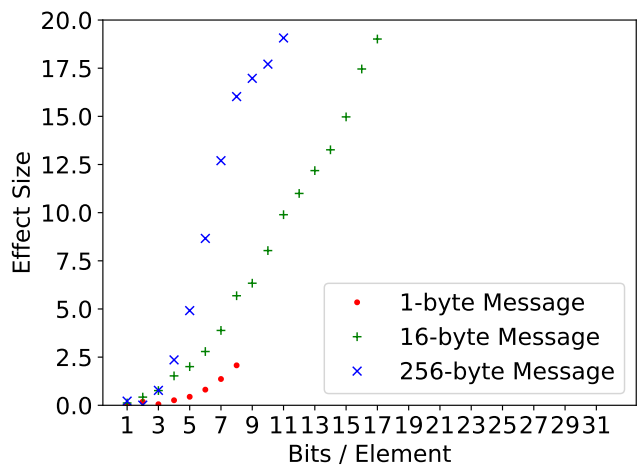

Figure 4.20: LZC Effect Sizes for TCP ISN

sizes for complexity tests were generally less than 0.01 as shown in Tables 4.9 to 4.11 . Figures 4.17 to 4.28 show that, like with IP ID, traces with more bits per element generally have higher effect sizes, but with such small values for 1-bit traces this is of little consequence. Accordingly, the resulting logistic regression detector shown in Table 4.8 was no better than chance.

The likely reason for this discrepancy is that TCP ISNs can be truly random-no vestigial need to be unique in order to reconstruct fragmented IP packets unlike IP 


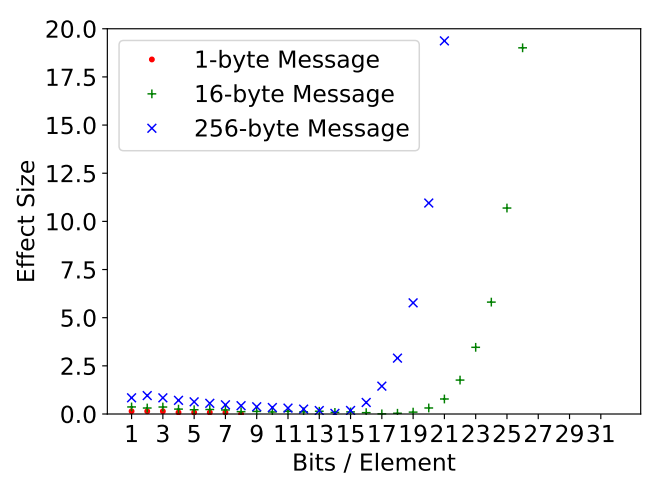

Figure 4.21: FOE Effect Sizes for TCP ISN

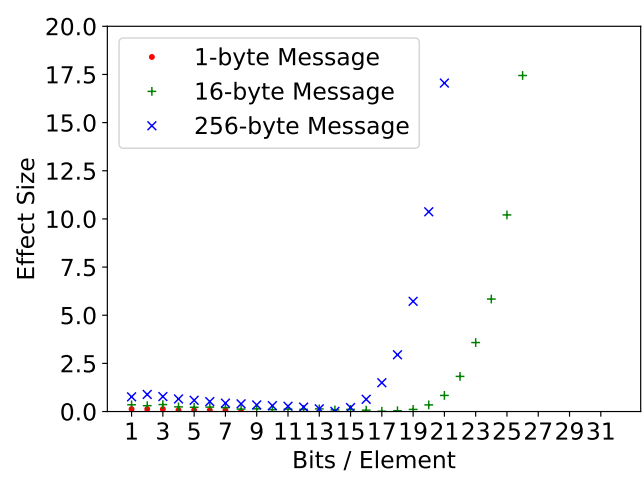

Figure 4.23: REP Effect Sizes for TCP ISN

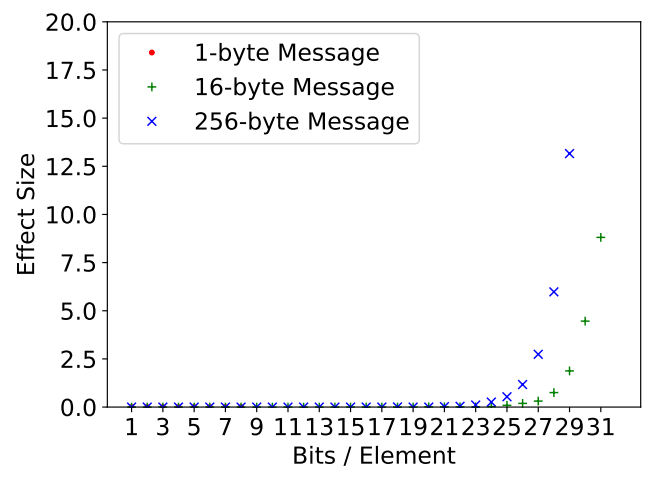

Figure 4.25: KS Effect Sizes for TCP ISN

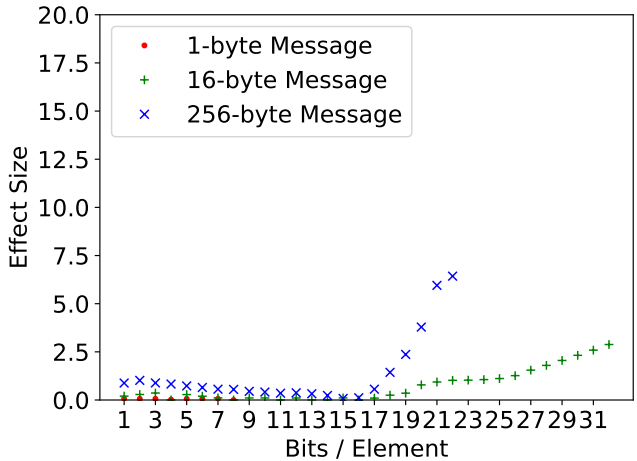

Figure 4.22: CCE Effect Sizes for TCP ISN

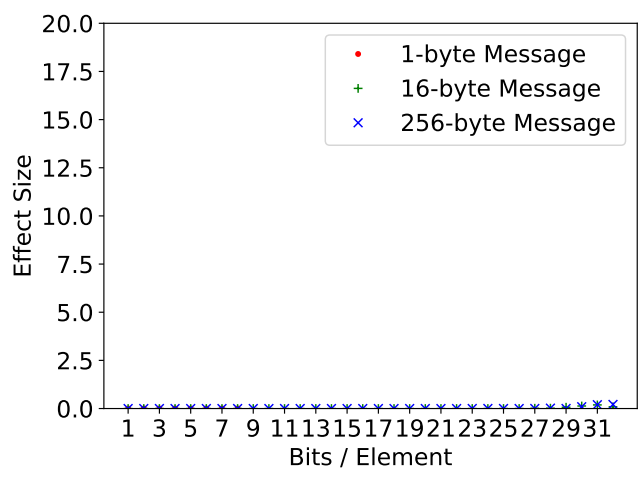

Figure 4.24: COV Effect Sizes for TCP ISN

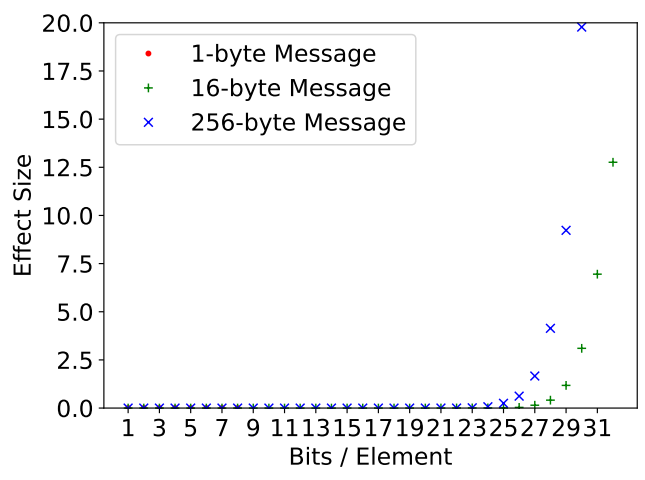

Figure 4.26: WCX Effect Sizes for TCP ISN 


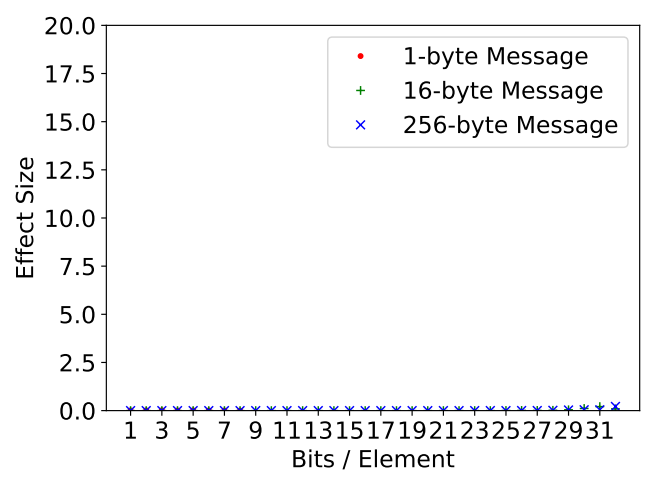

Figure 4.27: SPR Effect Sizes for TCP ISN

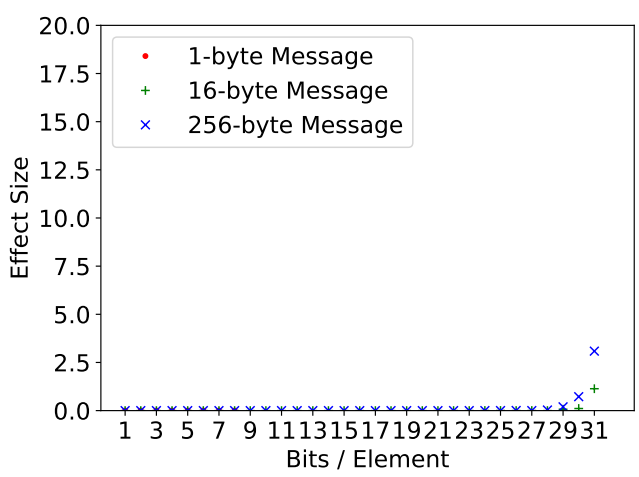

Figure 4.28: REG Effect Sizes for TCP ISN

ID. The small fragments of messages that we embed are also very close to random, so tests relying on information content cannot distinguish the two. This observation reveals something of a trade-off in how systems should choose their TCP ISNs. The conventional wisdom is that they should be truly random so as to make it difficult for an attacker to spoof a connection, but doing this creates an opportunity for a nearly undetectable covert channel (at least by the statistical tests considered in this chapter). That said, this is not much of a trade-off because almost any system will have much greater exposure from spoofed connections than from covert channels.

Despite this, there is still a reason why an attacker might use an IP ID covert channel and thus why one might want to detect them. Most obviously there is bandwidth: machines send out one IP ID per IP packet but only one TCP ISN per TCP connection and, since one TCP connection generally comprises many packets, many more IP IDs are transmitted than TCP ISNs. For instance, the dataset used in this chapter contains 76 times more IP IDs than TCP ISNs. Furthermore, using TCP ISN requires more access to the machine than IP IDs. With IP IDs an attacker can just change 
what IP IDs the machine sends and if the packet is never fragmented (the usual case) no one is likely to notice; with TCP ISN the attacker would not only have to change what is sent, but also what sequence number the machine expects to receive for subsequent segments.

\subsubsection{Repeating the Experiment for IP TTL}

The limited transferability to TCP ISN does not mean that the statistical tests evaluated in this chapter are specific to IP ID covert channels. IP Time-to-Live (TTL) is another network protocol field that can be used to build a covert channel, and was one of many studied by Gunadi and Zander [GZ17b]. IP TTL specifies the number of hops an IP packet can use to travel though a network (i.e., the number of routers it can pass through). At each hop the value in IP TTL is decremented and when it reaches zero the packet is dropped. This mitigates the impact of routing loops by preventing packets from cycling and taking up bandwidth forever. Of course this means that the value if IP TTL is regularly accessed and it's value is acted upon, which would seem to make it a poor choice for a covert channel. However, it's value is only impactful if it starts off low enough to reach zero before the packet arrives at it's destination. Modifying the value by a small amount will not affect the functioning of the system so long as the packets' initial IP TTL values are set high enough — with a margin of error - to not risk being mistakenly dropped in transit.

Using the same methodology as above, we trained a classifier to detect IP TTL covert channels. The results are presented in Table 4.12 and are similar to those for IP ID covert channels in that the accuracy of the detector remains high. On the other hand, 
the relative performance of each test was different; notably Repetition performed very poorly for all but the 4-byte messages. Seeing the effect size tables for IP TTL sheds some light onto this observation: Tables 4.13 to 4.15 as well as Figures 4.29 to 4.40 demonstrate that unlike IP ID for IP TTL it is the 8-bit traces that produce the smallest effect and are thus presumably hardest to classify. This is the case for every complexity test except for Repetition which continues to perform worst on 1-bit traces. Accordingly, Table 4.12 compares the other tests to Repetition at it's worst. Table 4.16 rectifies this by showing the performance of a detector on 8-bit traces. The tendency for Repetition to perform worse on longer messages continues, but only for 256-byte messages and for 4-byte messages Repetition performs the best while most other tests suffer markedly. That said, Lempel-Ziv Complexity performs well for all message sizes for both 8-bit and 1-bit traces, so that would be the test to use for a single test detector, especially since a practical detector would be need to work for any number of bits per packet without knowing what to expect ahead of time because whoever is using the covert channel can presumably change that at will. Combining tests is also useful for IP TTL as evidenced by the performance of the all-test detector which mostly matches that of Lempel-Ziv Complexity, but outperforms it on 8-bit traces with 4-byte message by leveraging Repetition's unmatched performance there. 
Table 4.12: Detector accuracy for IP TTL covert channel for various message sizes

\begin{tabular}{lllll}
\hline Message Size & 256 bytes & 64 bytes & 16 bytes & 4 bytes \\
\hline LZMA & $1 \pm 0.00039$ & $1 \pm 0.00075$ & $0.996 \pm 0.0021$ & $0.953 \pm 0.0072$ \\
LZ77 & $1 \pm 0$ & $0.999 \pm 0.00077$ & $0.998 \pm 0.0017$ & $0.947 \pm 0.0075$ \\
LZ78 & $1 \pm 0.00052$ & $0.999 \pm 0.00082$ & $0.997 \pm 0.0019$ & $0.968 \pm 0.0064$ \\
LZC & $1 \pm 0$ & $0.999 \pm 0.00077$ & $0.996 \pm 0.0022$ & $0.965 \pm 0.0063$ \\
FOE & $1 \pm 0$ & $1 \pm 0.00076$ & $0.998 \pm 0.0017$ & $0.992 \pm 0.0031$ \\
CCE & $1 \pm 0$ & $0.999 \pm 0.00077$ & $0.998 \pm 0.0016$ & $0.984 \pm 0.0045$ \\
REP & $0.504 \pm 0.056$ & $0.584 \pm 0.11$ & $0.78 \pm 0.025$ & $0.946 \pm 0.0078$ \\
All & $1 \pm 0$ & $1 \pm 0.00076$ & $0.997 \pm 0.0019$ & $0.981 \pm 0.0053$ \\
\hline
\end{tabular}

Table 4.13: Effect Sizes for 256-byte messages in an IP TTL covert channel

\begin{tabular}{lllllll}
\hline & 8-bit Trace & & Minimum & & Min. Index \\
\hline Encrypted? & $\mathrm{Y}$ & $\mathrm{N}$ & $\mathrm{Y}$ & $\mathrm{N}$ & $\mathrm{Y}$ & $\mathrm{N}$ \\
\hline LZMA & 1.04 & 3.32 & 1.04 & 3.32 & 8 & 8 \\
LZ77 & 1.13 & 1.46 & 1.13 & 1.46 & 8 & 8 \\
LZ78 & 2.14 & 0.594 & 2.14 & 0.594 & 8 & 8 \\
LZC & 0.00324 & 3.9 & 0.00324 & 3.9 & 8 & 8 \\
FOE & 2.36 & 0.118 & 2.36 & 0.118 & 8 & 8 \\
CCE & 0.114 & 3.9 & 0.114 & 3.9 & 8 & 8 \\
REP & 40.6 & 5.71 & 1.73 & 1.67 & 1 & 1 \\
COV & 6.17 & 2.97 & 0.00284 & 0.00203 & 1 & 1 \\
KS & 56.1 & 57.3 & 3.86 & 3.93 & 6 & 6 \\
WCX & 26.7 & 32.6 & 1.9 & 1.88 & 6 & 6 \\
SPR & 0.221 & 0.223 & 0.00383 & 0.00977 & 4 & 4 \\
REG & 1.87 & 1.14 & 0.025 & 0.0246 & 1 & 1 \\
\hline
\end{tabular}


Table 4.14: Effect Sizes for 16-byte messages in an IP TTL covert channel

\begin{tabular}{lllllll}
\hline & 8-bit Trace & & Minimum & & Min. Index \\
\hline Encrypted? & $\mathrm{Y}$ & $\mathrm{N}$ & $\mathrm{Y}$ & $\mathrm{N}$ & $\mathrm{Y}$ & $\mathrm{N}$ \\
\hline LZMA & 1.23 & 1.23 & 1.23 & 1.23 & 8 & 8 \\
LZ77 & 1.03 & 1.34 & 1.03 & 1.34 & 8 & 8 \\
LZ78 & 0.974 & 1.68 & 0.974 & 1.68 & 8 & 8 \\
LZC & 0.841 & 2.14 & 0.841 & 2.14 & 8 & 8 \\
FOE & 0.0901 & 0.439 & 0.0901 & 0.439 & 8 & 8 \\
CCE & 0.954 & 1.4 & 0.954 & 1.4 & 8 & 8 \\
REP & 11 & 6.95 & 2.55 & 2.55 & 1 & 1 \\
COV & 3.29 & 0.873 & 0.00194 & 0.000395 & 1 & 1 \\
KS & 22.8 & 23.6 & 1.51 & 1.64 & 6 & 6 \\
WCX & 10.5 & 13.6 & 0.52 & 0.546 & 6 & 6 \\
SPR & 0.135 & 0.136 & 0.00577 & 0.0173 & 4 & 4 \\
REG & 0.0971 & 0.0663 & 0.0948 & 0.0663 & 1 & 8 \\
\hline
\end{tabular}

Table 4.15: Effect Sizes for 1-byte messages in an IP TTL covert channel

\begin{tabular}{lllllll}
\hline & 8-bit Trace & \multicolumn{2}{r}{ Minimum } & \multicolumn{2}{r}{ Min. Index } \\
\hline Encrypted? & $\mathrm{Y}$ & $\mathrm{N}$ & $\mathrm{Y}$ & $\mathrm{N}$ & $\mathrm{Y}$ & $\mathrm{N}$ \\
\hline LZMA & 0.234 & 0.234 & 0.234 & 0.234 & 1 & 1 \\
LZ77 & 1.79 & 1.79 & 0.565 & 0.565 & 7 & 7 \\
LZ78 & 0.973 & 0.973 & 0.973 & 0.973 & 8 & 8 \\
LZC & 0.89 & 0.89 & 0.835 & 0.89 & 5 & 8 \\
FOE & 0.977 & 0.977 & 0.977 & 0.977 & 8 & 8 \\
CCE & 1.56 & 1.56 & 1.53 & 1.55 & 5 & 7 \\
REP & 0.317 & 0.317 & 0.317 & 0.317 & 8 & 8 \\
COV & 0.409 & 0.409 & $4.69 \mathrm{e}-05$ & 0.000422 & 3 & 3 \\
KS & 6.32 & 5.37 & 0.452 & 0.809 & 5 & 5 \\
WCX & 3.77 & 3.55 & 0.132 & 0.303 & 5 & 5 \\
SPR & 0.113 & 0.113 & 0.00138 & 0.00925 & 3 & 6 \\
REG & $\perp$ & $\perp$ & $\perp$ & $\perp$ & $\perp$ & $\perp$ \\
\hline
\end{tabular}


Table 4.16: Detector accuracy for IP TTL covert channel with 8-bit traces for various message sizes

\begin{tabular}{lllll}
\hline Message Size & 256 bytes & 64 bytes & 16 bytes & 4 bytes \\
\hline LZMA & $0.975 \pm 0.0072$ & $0.921 \pm 0.015$ & $0.897 \pm 0.028$ & $0.787 \pm 0.014$ \\
LZ77 & $0.924 \pm 0.0098$ & $0.906 \pm 0.01$ & $0.921 \pm 0.0092$ & $0.919 \pm 0.0096$ \\
LZ78 & $0.669 \pm 0.18$ & $0.636 \pm 0.18$ & $0.895 \pm 0.014$ & $0.854 \pm 0.012$ \\
LZC & $1 \pm 0$ & $1 \pm 0.00097$ & $0.986 \pm 0.0065$ & $0.936 \pm 0.0082$ \\
FOE & $0.562 \pm 0.22$ & $0.497 \pm 0.14$ & $0.776 \pm 0.019$ & $0.836 \pm 0.014$ \\
CCE & $0.998 \pm 0.0017$ & $0.976 \pm 0.0062$ & $0.897 \pm 0.013$ & $0.810 \pm 0.019$ \\
REP & $0.746 \pm 0.24$ & $1 \pm 0.00067$ & $0.997 \pm 0.0019$ & $0.955 \pm 0.007$ \\
All & $1 \pm 0$ & $1 \pm 0.00087$ & $0.988 \pm 0.0039$ & $0.942 \pm 0.0088$ \\
\hline
\end{tabular}

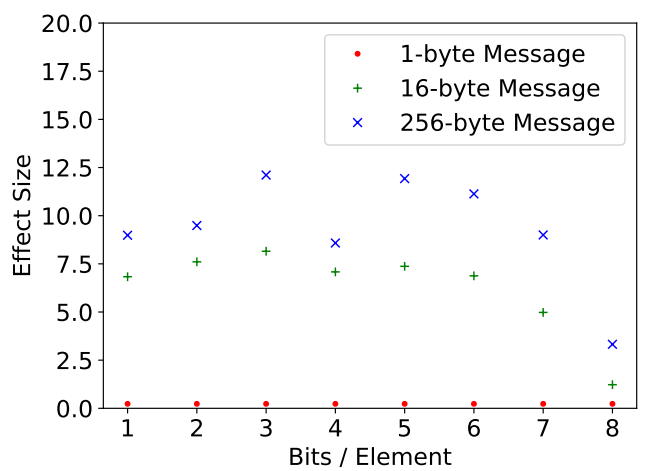

Figure 4.29: LZMA Effect Sizes for IP TTL

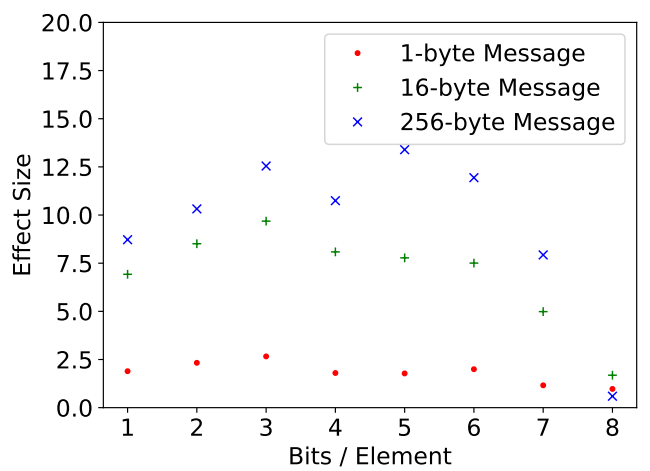

Figure 4.31: LZ78 Effect Sizes for IP TTL

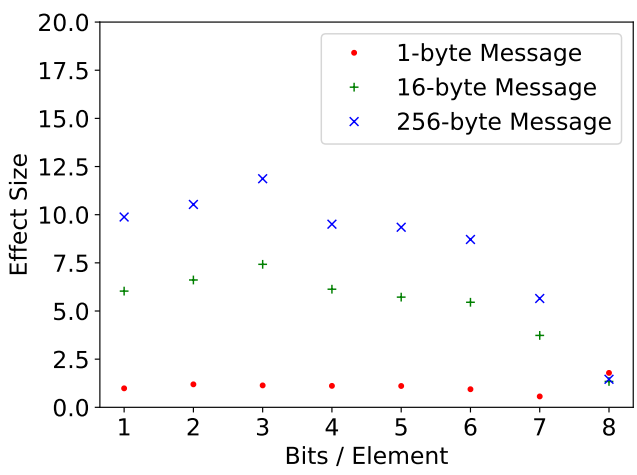

Figure 4.30: LZ77 Effect Sizes for IP TTL

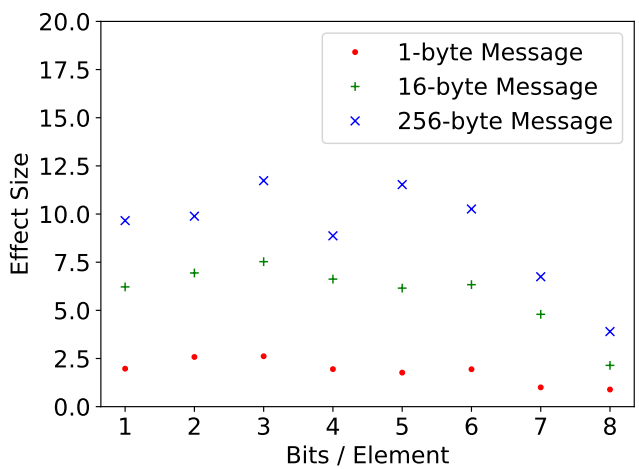

Figure 4.32: LZC Effect Sizes for IP TTL 


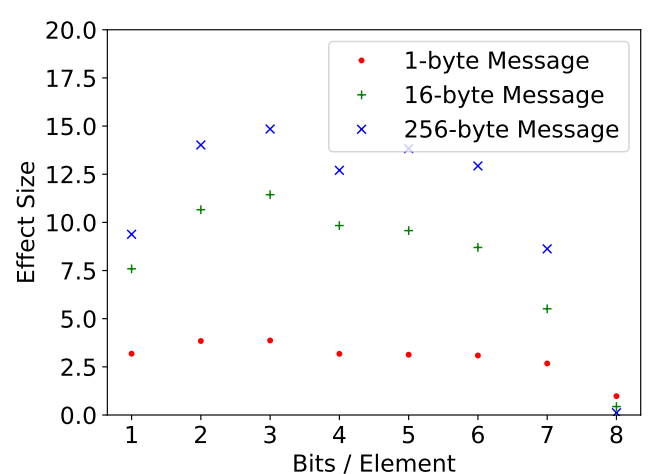

Figure 4.33: FOE Effect Sizes for IP TTL

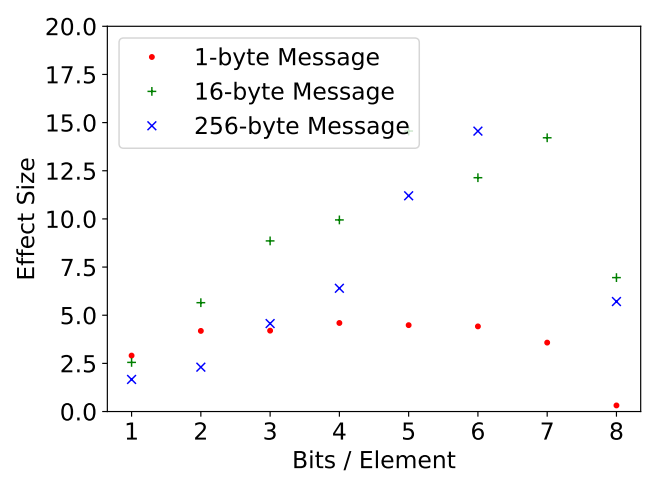

Figure 4.35: REP Effect Sizes for IP TTL

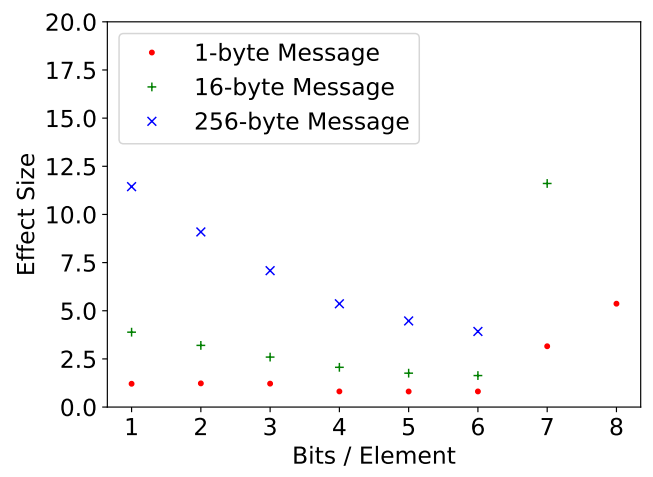

Figure 4.37: KS Effect Sizes for IP TTL

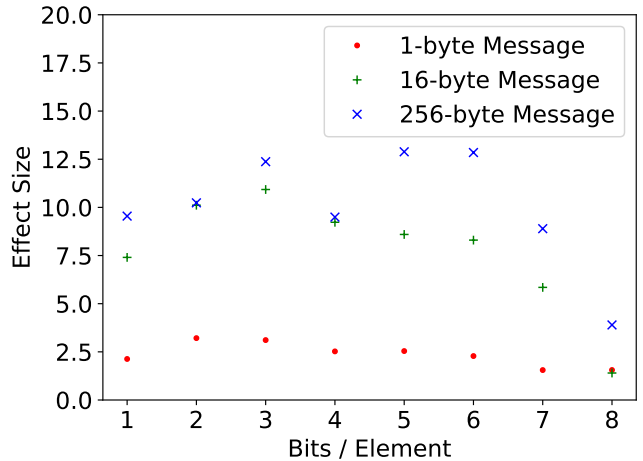

Figure 4.34: CCE Effect Sizes for IP TTL

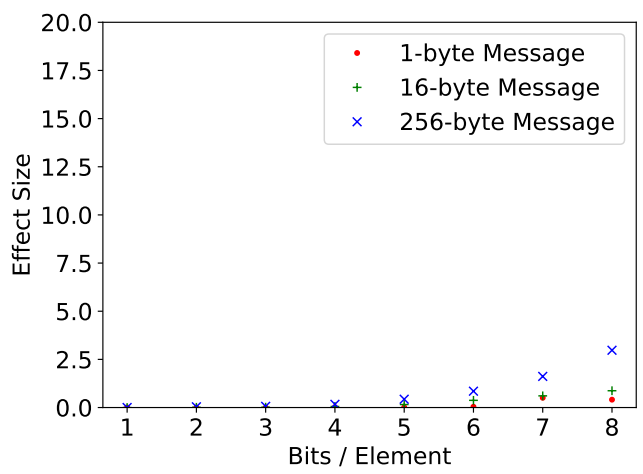

Figure 4.36: COV Effect Sizes for IP TTL

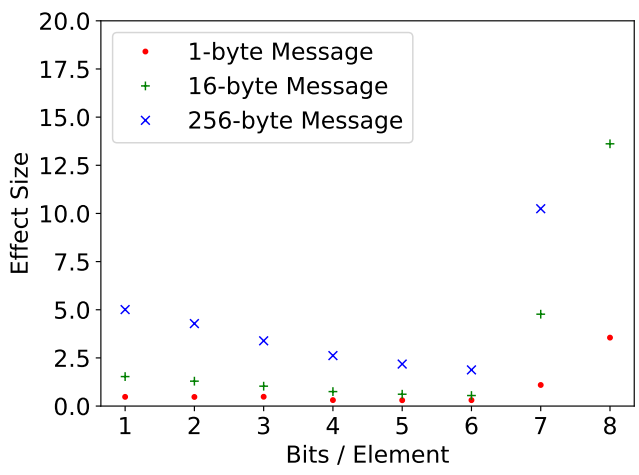

Figure 4.38: WCX Effect Sizes for IP TTL 


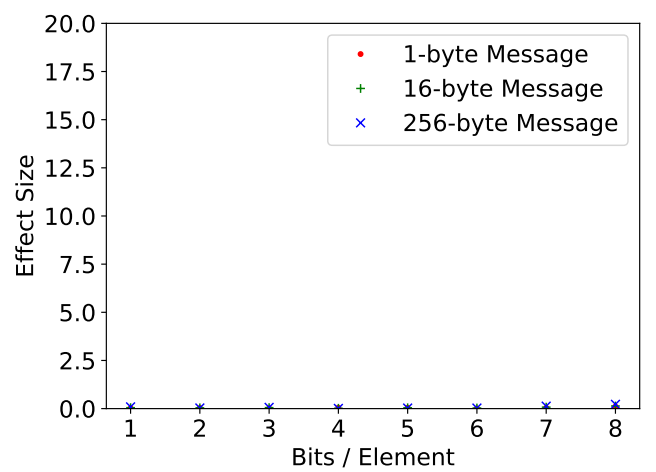

Figure 4.39: SPR Effect Sizes for IP TTL

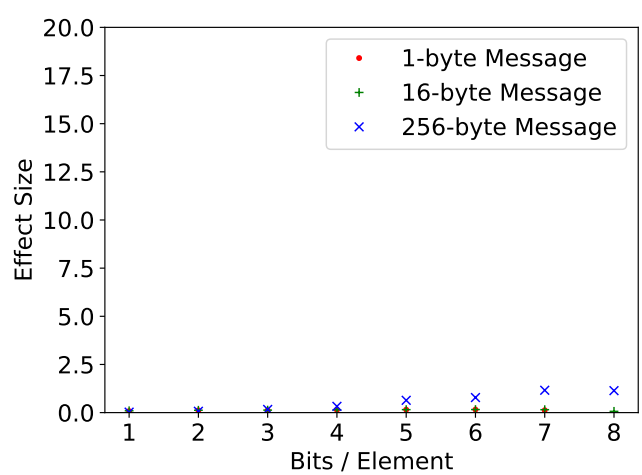

Figure 4.40: REG Effect Sizes for IP TTL

\subsection{Discussion}

This chapter has evaluated the effectiveness and applicability of several statistical tests to detect storage-based covert channels. The tests were selected based on their past success in being effective to detect timing-based covert channels. More specifically, we conducted several experiments on sequences of IP IDs, IP TTLs and TCP ISNs with and without information embedded into them. The results of the experiments show that complexity tests are much more effective that distributional tests for detecting storage-based covert channels. Repetition in particular was extremely effective for such a simple test. Many of the tests were determined to be able to detect covert channels on their own and combining multiple tests into a multi-dimensional classifier did not bring any significant improvement which means that simple covert channel detectors can be built using a single test. 


\section{Chapter 5}

\section{Implementation}

One of the primary goals of this project was to build a system to detect covert channels in real-time by analysing ethernet communications. Chapter 4 detailed the process for deciding which techniques ought to be used for a good covert channel detector. Using those results, this chapter describes the design and implementation of such a detector. The system has been dubbed SEACCOW which stands for Security Evaluation and Assurance Covert Channel Online Warden. The name is derived from the Cyber Security Evaluation and Assurance (CyberSEA) Research Lab in which it was developed as well as its purpose. The code for SEACCOW is available at: gitlab.com/CyberSEA-Public/SEACCOW.

The system was built using a Field Programmable Gate Array (FPGA) which is a computing technology that bridges the gap between hardware and software. Like software, it is quickly and easily reprogrammable, but otherwise it behaves like hardware in that computations are performed by connecting gates and storing data in registers 
in time with clock edges rather than by executing a sequence of instructions. FPGAs have both hard and soft components. Hard components are implemented like regular hardware; they are fast and small (i.e. that do not use up much area on a intergrated circuit), but their functionality is fixed. The hard components built into FPGAs are typically circuits that are useful in a broad swath of applications such as memories and multipliers. Soft components are what differentiate FPGAs from regular hardware; their functonality can be reprogrammed in minutes, but this comes at a cost in terms of speed and area. Soft components are still fast and efficient compared to software, but not as much as regular hardware.

Section 5.1 begins this chapter by describing the various vendor-specific components that were needed for this implementation. Section 5.2 follows with a catalogue of the limited operations performed in software. Section 5.3 forms the bulk of the chapter and describes the portable hardware modules that were written to detect covert channels. Finally, Section 5.4 presents the performance of the system.

\subsection{Non-portable Components}

SEACCOW was conceived as a generic and broadly-useful platform for detecting storage covert channels in real-time using FPGAs. As such, the core of its design consists of several modules written in the SystemVerilog Hardware Description Language. Nonetheless, several aspects of the design had to be adapted to the available hardware and are thus are not trivially portable. 


\subsubsection{Board}

SEACCOW was tested on a Terasic DE2-115 development board [Ter17]. This was chosen primarily because it is one of the few FPGA development boards that comes with two ethernet ports built-in. The FPGA installed on the board is an Intel (whose FPGA business was formerly known as Altera) Cyclone IV E device [Alt16]. The Cyclone family of FPGAs are low-cost devices whose fourth generation first appeared in 2009. 'E' designated variants have none of the special hard components that other Cyclone IV devices have. Besides the FPGA, the only other board components that are critical to SEACCOW's functionality are the two Marvell 88E1111 Ethernet PHY chips that directly observe and control the signals on the ethernet cables. Figure 5.1 is a picture of the board with all these key features identified.

\subsubsection{Ethernet Core}

SEACCOW needs to receive and transmit Ethernet frames. This was done using Intel's Triple-Speed Ethernet IP (Intellectual Property, not Internet Protocol) that translates between ethernet communication and a simple Avalon streaming interface [Int19] that allows data to be transmitted easily between SEACCOW's various modules. 


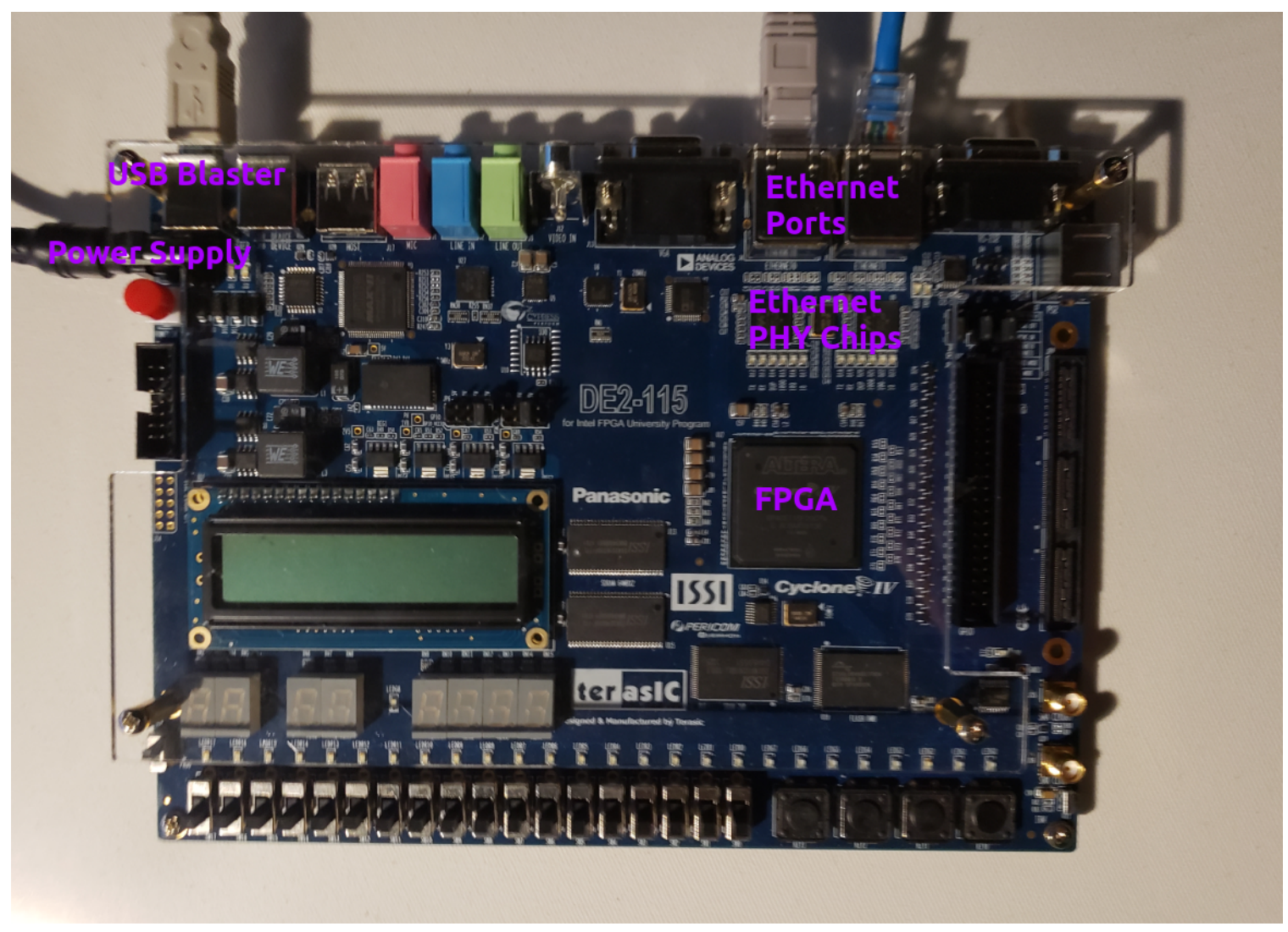

Figure 5.1: DE2-115 Development Board 


\subsubsection{Nios II Processor}

A Nios II soft processor is needed to initialize the configuration registers of the Ethernet Cores and the Ethernet PHY chips.

\subsubsection{Phase Locked Loop}

The FPGA's Phase Locked Loop hardware and Intel's design tools were used to generate the various clocks that are needed by the system. The Ethernet cores require several clocks running at different frequencies for their internal operation; the triplespeed ethernet cores can operate at three different data rates each of which requires the core to run at a different frequency which requires a different clock. The rest of the system (i.e. all the components that were developed specifically for SEACCOW) all runs on a single clock.

\subsubsection{Top-level Module}

The top-level module of a hardware design is similar to the 'main' function of a software program. It is the starting point for all functionality and generally handles the interactions of the design with the outside world. As such, the top-level module connects SEACCOW's internal functionality (which is managed by the SEACCOW Internal module, see Section 5.3.1) and the board hardware. 


\subsection{Software}

The software, written in C, runs on the Nios II processor and initializes the Ethernet cores and PHYs. For the Ethernet PHYs it:

1. Enables automatic crossover, so that crossover ethernet cables need not be used.

2. Sets up delays for the input and output clocks so that the signals on the ether cables are sampled at roughly the optimal phase.

As for the internal Ethernet cores it:

1. Enables receiving and transmitting.

2. Sets the speed to one gigabit per second.

3. Enables promiscuous mode, so that the core will send all frames to the main system regardless of what MAC address they were sent to.

4. Turns on $\mathrm{CRC}^{1}$ forwarding to so that the same CRC can be transmitted without having to recalculate it.

5. Instructs the core to drop error frames; for simplicity.

6. Instructs the core to omit CRC calculation so as to retransmit the frames it receives unchanged.

\footnotetext{
${ }^{1}$ Cyclic redundancy check, an error detection field used by Ethernet.
} 


\subsection{Hardware}

The bulk of SEACCOW is implemented as FPGA components described using SystemVerilog. The many modules that form this implementation are described in this section. Each subsection corresponds one-to-one to a module in the SystemVerilog implementation and vice-versa.

\subsubsection{SEACCOW Internal}

This module acts like a top-level module for SEACCOW internal modules: it instantiates the other modules and provides the logic needed to connect them. It is instantiated twice by the top-level module - once for each direction, machine-to-network and network-to-machine - along with the necessary board-specific and otherwise nonportable modules.

\subsubsection{FIFO}

Upon receiving ethernet frames, SEACCOW stores them in a first-in-first-out queue. Its function is to introduce a delay between receipt and transmission so that the main covert channel detection modules have time to process the frames and can decide whether to drop and/or store a frame. 


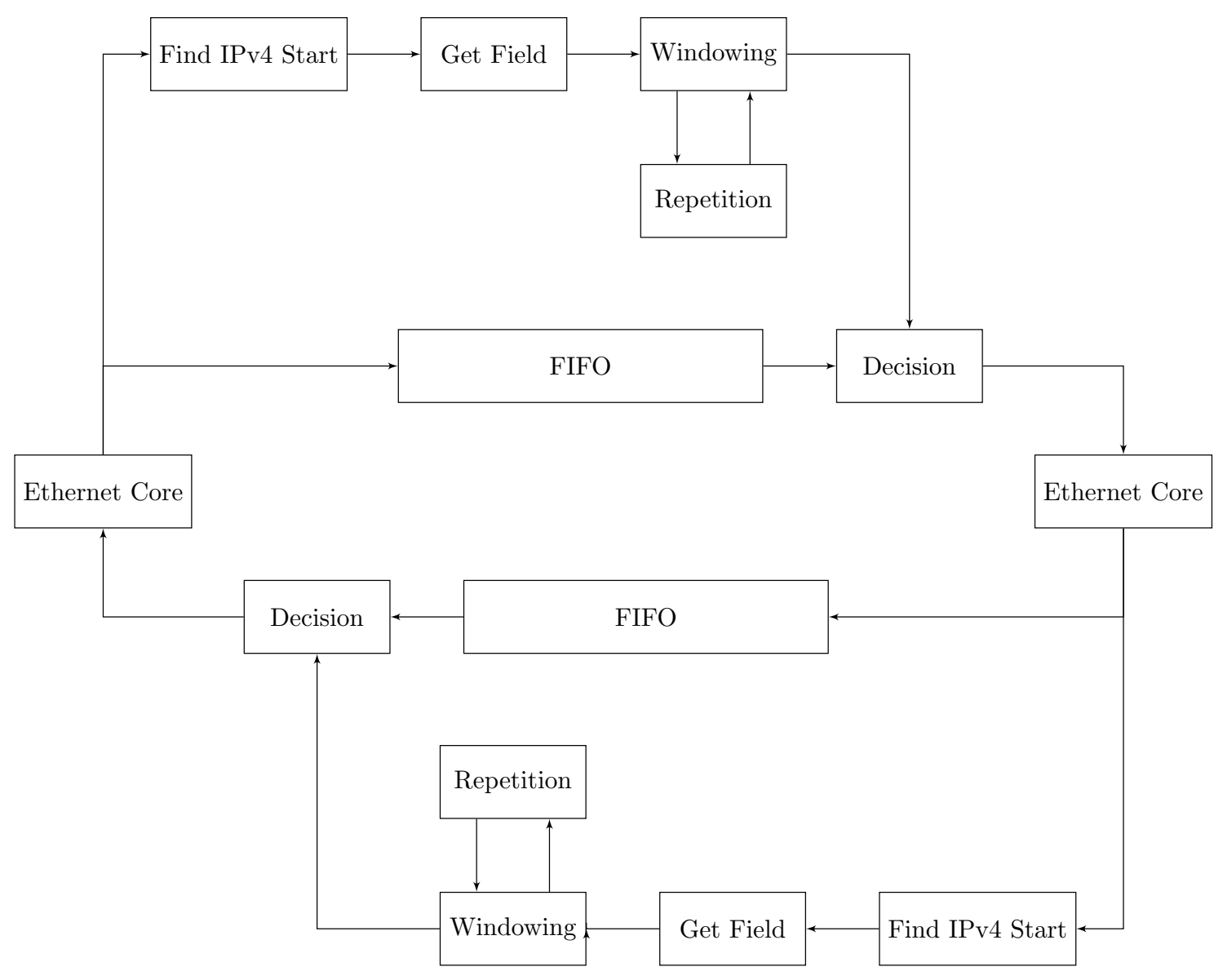

Figure 5.2: Block Diagram of Internal Modules 


\subsubsection{Find IPv4 Start}

As its name suggests, the purpose of this module is to find the start of IPv4 packet headers within the ethernet frames. It outputs a signal that usually stays low and goes high for one cycle whenever the start of a IPv4 header is detected. It is assumed that ethernet frames and IPv4 packets are mapped one-to-one, i.e., each frame contains exactly one packet, and that the IPv4 Header immediately follows the ethernet header; this is standard practice. The module handles VLAN-tagged frames, correctly skipping the location the VLAN tags location to find the end of the ethernet header at its shifted location. Other types of ethernet frame (as defined by the Ethertype field) are not handled and are ignored because they do not constitute a substantial portion of the packets in the dataset used to evaluate covert channel detection techniques [Gar17].

\subsubsection{Get Field}

This module extracts an arbitrary field from the ethernet frames. The field can be of any size and is defined by its distance from a 'start' signal such as the output of the Find IPv4 Start module. This is used to extract IP Identification but could be easily repurposed to extract any other IP Header field or even a header field from another protocol if it is provided with a 'start' signal for that protocol's header. Each instantiation of the module must be provided with three parameter values: a field size, a word index and an offset. The field size is the number of bits in the field that should be extracted; the word index is the number of 32-bit words that need to be 


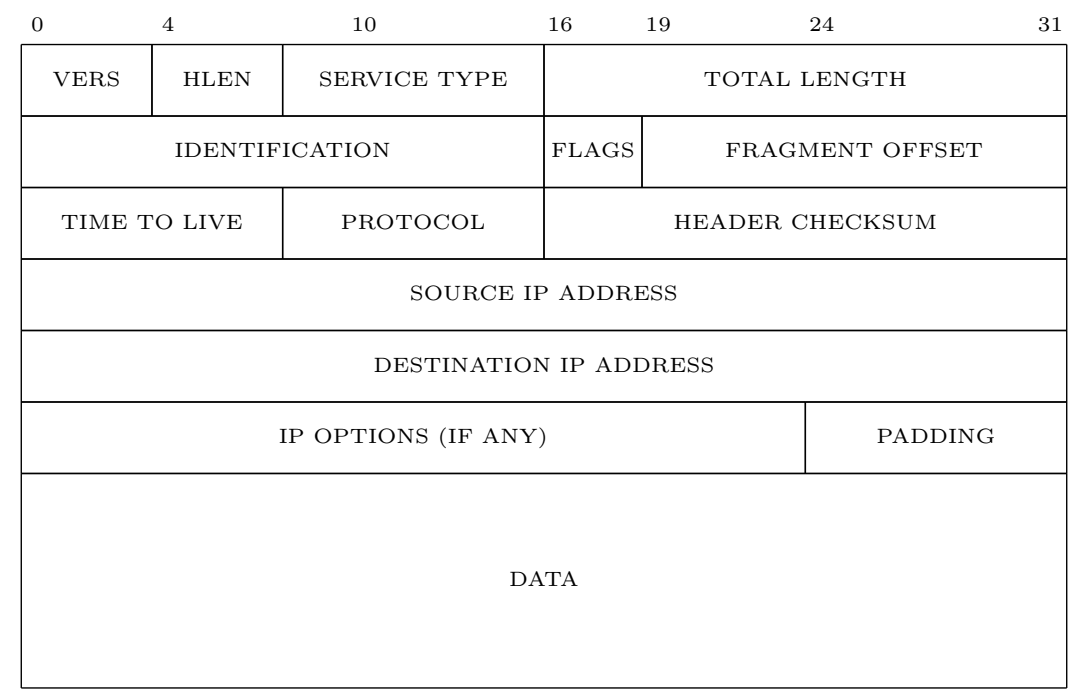

Figure 5.3: IPv4 Header Format

ignored after receiving a 'start' signal before extracting the field and the offset is the number of bits from the most-significant bit to the start of the field to be extracted. In order to extract the IP Identification field, the module must be instantiated with a field size of 16, a words index of 1 and an offset of 0 . If, on the other hand, one wanted to extract the IP Flags one would use a field size of 4, a words index of 1 and an offset of 16. This is illustrated in Figure 5.3 which shows the format of an IPv4 Header; the word index corresponds to the row that contains the field to extract, the offset is the column where the field begins and the field size is the number of columns taken up by the field.

\subsubsection{Count Words}

A helper module for Find IPv4 Start and Get Field that counts 32-bit words received from the Ethernet controllers. 


\subsubsection{Repetition}

The purpose of this module is to count the number of repeated entries in a stream of data. More specifically it takes as input the fields extracted by the Get Field module and counts how many of the values extracted are equal to other values extracted. This count, as well as the module's knowledge of which values have been seen (and hence which are repeats), can be cleared by raising the clear signal for one clock cycle. The remaining inputs are the clock, the reset and a valid signal to indicate the value driven on the field input should be considered.

The main feature of this module is its ability to clear in a single clock cycle. Since it is generally impossible to clear a whole memory in a single cycle, achieving this meant one of two things: the module's knowledge of preceding field values had to be stored somewhere besides a memory — effectively this implies storing it in registers - or using some non-trivial design of repetition counter. Using registers to store history would be grossly inefficient because each bit stored in a register takes up far more area than it would in a memory. So, a repetition counter that trades off extra memory utilization and small chance of inaccuracy for instantaneous clearing was devised.

The previous paragraph made reference to a trivial repetition counter and it's limitations. In order to elucidate that, and since it will constitute the main conceptual building block of the actual repetition module, such a module is described below. 


\section{Trivial Repetition Module}

The simplest way to build a repetition module for a stream of $N$-bit numbers is to instantiate a memory with an $N$-bit address size and a single bit of storage at each address. When each new number is received, we can read the value it addresses and store a ' 1 ' in its place. We then increment a counter if the value read is equal to ' 1 '. As long as the contents of the memory was all zero at the start of the stream this procedure correctly computes the number of repeated values.

\section{Clearing Optimization}

The single-cycle clearing optimization works by using multiple memory blocks instead of just one. At any given time one memory block is used to keep track of repetitions while the others are being cleared. When a 'clear' signal is received the module swaps out the memory module it has been using and starts using another. If the new memory block is all zero when it starts being used, the module will work just like the Trivial Repetition Module. Clearing of unused memories is still not instantaneous - only one bit can be cleared per clock cycle - so depending on the number of memories used, their size ( $\left(2^{L}\right.$ where $L$ is the size of each datapoint), the clearing frequency and the rate at which the module receives valid data the repetition count may not be correct. That said, the clearing frequency and the maximum rate at which new datapoints arrive is quite predictable and the number of memories used can be adjusted accordingly. Specifically, the number of memories should be greater than the memory size divided by the number of clock cycles between datapoints multiplied the number of datapoints between clears. That is, 


$$
M>\frac{S}{C D}
$$

where $M$ is the number of memories, $S$ is the memory size, $C$ is the number of clock cycles per datapoint and $D$ is the number of datapoints per clear. Satisfying this constraint ensures that there will be enough clock cycles after the use of a memory for it to be fully cleared before being used again. This can be achieved easily enough, thus ensuring the correct functioning of a Repetition module designed in this way.

\subsubsection{Windowing}

In order to search through a stream of data for covert channel communication we need to split the stream in chunks that can be assessed independently. If a stream starts being used as a covert channel we do not want a low repetition rate earlier in the stream to preclude the covert information flow being detected. Similarly, if a stream stops containing any covert information, the detector should be able to identify this and let the packets pass through without interference. Moreover, if the repetition test is to work by flagging a series of packets based on its repetition rate it needs a denominator to compute said rate. A simple way to achieve this is to split the stream into fixed size chunks and clear the repetition module at the start of each of these windows.

The size of these windows is governed by a few considerations. First and foremost, the window size must be long enough that a repetition detector will able to reliably identify a covert message if it exists. This length was experimentally determined by 
the software testing described in Chapter 4. A four-byte message with one bit of message in each field was detectable by the repetition test; this translates to a 32 element window ( 4 bytes $\times 8$ bits per bytes $\times 1$ bit per element). Furthermore, the windows ought not to be much larger than that minimum size so that the detector can react to a change in the presence of a covert message soon after it occurs. Ideally, the window size should also be a power of two. This makes the module slightly more efficient (the counter can just roll over to zero) and also means that the count produced by the repetition module can be interpreted as repetition rate. More specifically, for a window of size $2^{X}$ we conceptually introduce a radix point between the $X^{\text {th }}$ and $X+1^{\text {th }}$ least significant bits of the count to get the fraction of elements that are repeated. Given this, a 32 element window seems to be the right choice.

To sum up, what the windowing module does is ultimately quite simple, it instantiates the repetition module and clears it after every 32 valid fields have been received. It also takes the repetition count produced by the repetition module and compares to an empirically determined threshold (see Section 5.4), thus deciding whether or not to flag a chunk of packets as containing a covert message.

\subsubsection{Decision}

The previous modules are concerned with detecting the presence of covert information. This module, on the other hand, handles what to do with the underlying packet once a covert channel has been detected in its header. The goal is to block the transfer of a covert message by dropping the packets in which such information is detected and the bulk of the logic in this module is used to coordinate between the time when 
the windowing module indicates that a covert channel exists (soon after the packet enters the FIFO) and when the packet leaves the FIFO to be transmitted back onto the ethernet network.

The basic design is to have two counters, one that counts frames as they enter the FIFO (the ingress counter) and another that counts them as they exit (the egress counter). These counters are paired with a memory block that stores whether each window is found to contain covert information. Since windows are just 32 packet sections of the stream of data, they can be identified by the counter values with their 5 least significant bits removed. These truncated counters can be used to address the memory; whenever a covert channel is detected, a ' 1 ' is written to the memory location corresponding to the window in which it was found. When the packets that belong to that window exit the FIFO the ' 1 ' is read and packets are not sent.

Coordinating the egress counter with the packets exiting the FIFO is trivial. We simply delay the words of the exiting packets by one cycle to allow for the memory to be checked. The coordination between the ingress counter and the detection of a covert channel, on the other hand, is not. The process for detecting a covert channel explained in Subsections 5.3.3 to 5.3.7 takes time and packets can be of arbitrary length so it is possible that by the time a covert channel is detected the ingress counter will have incremented one or more times due to a new packet starting. This issue is solved by taking snapshots of the ingress counter at the time of specific events so that a counter value mismatch cannot occur. The first such snapshot is when the Find IPv4 Start module detects the start of an IP packet. This happens within one cycle of the start of the packet, so the ingress counter cannot have already incremented 
before the snapshot is saved. This snapshot will remain the same until another IPv4 packet starts. Next, we take a snapshot of the IPv4 start snapshot whenever an IP ID field is extracted from the header. The design of the Repetition module is such that it will detect a covert channel 4 cycles after it gets a new IP ID value. If the sender is following protocol, it is not possible to finish sending the IP header, start a new packet and send a new IP ID within 4 cycles because the remaining mandatory portions of the current IP header (source and destination address, 4 bytes each) and the ethernet header of the next frame must contain more than 16 bytes. The system receives at most 4 bytes in each clock cycles, so receiving 16 bytes will take more than 4 clock cycle. Thus, if we were to assume that this detector would always work with protocol compliant traffic we could just use the IP ID extraction snapshot to address the memory and store a flag to indicate whether covert information has been detected in the corresponding window. That said, this compliance assumption is one we would prefer not to rely upon. Seeing as 4 extra registers is not a large expense, we can simply store a new snapshot of the IP ID extraction snapshot at every clock cycle until the time when the covert channel may be detected. The last of these can then confidently be used to identify the window in which a covert channel is being detected.

One final requirement for the decision module is that it must always either set the memory location addressed by the ingress counter (and it's snapshots) either to ' 1 ' or reset it to '0'. Setting to ' 1 ' is explained above: 4 cycles after the IP ID is extracted the memory location is ' 1 ' if the windowing module is signalling a covert channel at that moment, otherwise the memory location is reset to ' 0 '. However, this only covers that case is which an IP ID is actually found. The memory location must be 
cleared regardless of whether the frame contains an IP packet because the ingress counter will eventually roll over and we do not want to drop a frame based on a covert channel detected in a prior frame. To satisfy this requirement, we just reset the memory location to ' 0 ' as soon a new frame starts before we know if it contains a covert channel.

\subsubsection{Repetition Test Bench}

Among the modules previously described in this chapter, the Repetition module stands out as having the most complex behaviour. Because of this, it is more likely to have errors when initially written and far more likely to have errors that are hard to detect. This justifies a special test bench module to test the behaviour of the repetition module.

Even though the module uses a somewhat complex algorithm, the relation between its input and its output is simple in that the output is just a count of the repetitions in the input. This means that the test bench too can be quite simple: just take some data and pass it through the module and check that the output is correct. This is

all the Repetition Test Bench does; it reads a file and passes it to an instantiation of the repetition module as if is each line were an IP ID observed on the wire. The computed number of repetitions outputted by the module is checked against correct answer computed in software. The files being checked themselves are also generated in software. 


\subsubsection{SEACCOW Internal Test Bench}

Besides the repetition module, the other aspect of SEACCOW that really needs a test bench is the full integrated system. This includes all the modules that are internal to the FPGA (i.e., those that are not the top-level module and do not directly interact with the world outside the chip); together they take input from one ethernet controller and send out packets on the other. What the system test bench does is feed frames to the SEACCOW Internal module as if it were one of the ethernet cores and check what frames come out. The IP ID fields of the frames can be crafted such that some specific packets will be dropped if the system is working correctly.

\subsection{Performance}

In evaluating SEACCOW's performance two factors are of paramount importance: the accuracy with which covert channels are detected and the latency between covert information transfer and detection.

\subsubsection{Accuracy}

The accuracy of a covert channel detector is the fraction of windows that are correctly identified as containing or not containing a covert message. This will depend on how data is split into windows and the ways in which the message is embedded as well as other minutiae. Many such considerations were explored and tested in Chapter 4. Moreover, seeing as the hardware implementation described here operates 
Table 5.1: SEACCOW Confusion Matrix

\begin{tabular}{c|c|c|c}
\multicolumn{2}{c|}{} & \multicolumn{2}{c}{ Actual Class } \\
\cline { 3 - 4 } \multicolumn{2}{c|}{} & Positive & Negative \\
\hline $\begin{array}{c}\text { Predicted } \\
\text { Class }\end{array}$ & Positive & 979 & 7 \\
\cline { 3 - 4 } & Negative & 21 & 993
\end{tabular}

identically — using the same algorithm - to the software simulations of the previous chapter, there is good reason to believe that both must have the same accuracy. Thus, SEACCOW's accuracy for both IP ID and IP TTL covert channels should be roughly $95 \%$, corresponding to what was reported for a repetition detector on 4 byte messages (a 4 byte message is equivalent to the 32 element window size chosen in Subsection 5.3 .7 because 4 bytes contain 32 bits).

In order to verify these numbers, 1000 positive samples and 1000 negative samples were fed into SEACCOW. The threshold to flag a window as containing a covert message was set to 2 or more repetitions based on the software tests presented in Chapter 4. More specifically, 2 is the closest integer to the repetition rate that the logistic regression detector presented in Section 4.3.3 would predict has covert information with a probability of $50 \%$, thus the threshold between the windows that are more likely than not to contain a covert message and those that are probably benign. Of the 1000 positive samples 979 were correctly identified and of the 1000 negative samples only 7 were falsely identified; this same data is presented in the form of a confusion matrix in Table 5.1. The resulting accuracy is $98.6 \%$ which is consistent with the results reported in Table 4.7.

The only relevant potential discrepancy that could have existed between the behaviour of the SEACCOW hardware and the software simulation is the clearing optimization 
of the repetition module. If its constraints are violated, then the number of repetitions reported could be wrong and thus different from what the software implementation computes. However, the current implementation is such that number of clock cycles per datapoint is always large and the aforementioned constraints always satisfied.

\section{Class Imbalance}

The balanced set of inputs presented in Table 5.1 is not a very realistic scenario. Any real network will transmit far more negative samples than positive ones. However, it is inherent in the system's design that class imbalance cannot meaningfully affect the predictions. The threshold for the number of repetitions that triggers a positive prediction remains fixed and the calculation of the number of predictions is totally independent between windows, thus the prediction for one window cannot affect those for another window. In fact, since in the balanced case the false negatives outnumber the false positives, a more realistic scenario ought to have higher accuracy because the negative samples, which were correctly identified more often than positive ones, form a larger proportion of the dataset. Nevertheless, in the spirit of completeness, a confusion matrix for a scenario in which negative samples outnumber positives by two orders of magnitude is presented in Table 5.2. The overall accuracy is $97.75 \%$. The fact the false negative rate increased and the false positive rate decreased relative to the balanced case is attributable to chance. 
Table 5.2: SEACCOW Confusion Matrix with Class Imbalance

\begin{tabular}{c|c|c|c}
\multicolumn{2}{c|}{} & \multicolumn{2}{c}{ Actual Class } \\
\cline { 3 - 4 } \multicolumn{2}{c|}{} & Positive & Negative \\
\hline $\begin{array}{c}\text { Predicted } \\
\text { Class }\end{array}$ & Positive & 20 & 45 \\
\cline { 2 - 4 } & Negative & 0 & 1935
\end{tabular}

Table 5.3: SEACCOW Confusion Matrix with Sub-optimal Threshold

\begin{tabular}{c|c|c|c}
\multicolumn{2}{c|}{} & \multicolumn{2}{c}{ Actual Class } \\
\cline { 3 - 4 } \multicolumn{2}{c|}{} & Positive & Negative \\
\hline $\begin{array}{c}\text { Predicted } \\
\text { Class }\end{array}$ & Positive & 888 & 1 \\
\cline { 2 - 4 } & Negative & 112 & 999
\end{tabular}

\section{Threshold Setting}

The specific threshold of 2 repetitions used to predict the use of a covert channel is clearly a function of the underlying dataset that was used in Chapter 4. Notably, a different set of network traffic captures could have started off with a different repetition rate of IP IDs and that could change the optimal threshold value. Potential users of SEACCOW would be wise to periodically capture samples from their network and perform the analysis presented in Section 4.3.3 so as to ascertain any need for the threshold to be changed. That said, the large effect sizes yielded by the repetition test in Section 4.3.1 suggest that the optimal threshold should not be very sensitive to changes in the dataset. Furthermore, changing the detection threshold from 2 to 3 does not lead to a catastrophic decline is SEACCOW's accuracy. As shown in Table 5.3, SEACCOW still detects the presence of covert information with $94.35 \%$ of the time with a sub-optimal threshold. 


\subsubsection{Latency}

The rationale for having created a hardware implementation was to minimize latency and detect covert channels in real-time. As discussed in Section 5.3.8, the Repetition module takes 4 clock cycles to detect the presence of a covert channel. To this we add 1 cycle to extract the IP ID field get the 5 cycle latency SEACCOW needs to detect a covert channel. At a clock speed of $100 \mathrm{MHz}$, which SEACCOW achieves easily on an FPGA from 2009, this amounts to a latency of only 50 ns. For comparison, this is less than 4 times the amount of time an ordinary computer from the same era would take to do a single RAM access [Mic06].

To be more precise, recall that SEACCOW detects a covert channel when the number of repeated IP ID values in a 32 packet windows crosses a given threshold. The 5 clock cycle delay is between the moment the IP ID value that would put the window over the threshold exits the receiving Ethernet Core and enters the SEACCOW Internal module and when the system reports that has detected a covert channel being used.

\subsection{Concluding Remarks}

To sum up, SEACCOW successfully replicates the covert channel detection ability of the software simulations of Chapter 4. As a hardware implementation written in SystemVerilog, SEACCOW's design is somewhat complex because it must handle some intricate details of memory management and signal timing. What is gained from this is a much improved latency, easily good enough for a real-time system. 


\section{Chapter 6}

\section{Conclusion}

This thesis chronicles an investigation into the real-time detection of storage covert channels. The study of covert channels is not a new field. A substantial literature has been built up over the years to form a lofty foundation for projects such as this. This literature includes significant work on the effective construction of covert channels and the inspection of systems for potential covert communication, but what is most important to this project is the study of covert channel detection. A high-level sketch of the literature of that topic in provided in Chapter 2.

A clear take-away from Chapter 2 is that detection techniques for protocol-based covert channels are built on statistical tests applied to the data transmitted via said

protocol. Chapter 3 focuses on these tests, their history, the specifics of their functioning and their applicability —or lack thereof - to this project's goals.

Chapter 4 continues where Chapter 3 leaves off, describing the experiment that was 
used to evaluate the suitability of each test to a real-time storage covert channel detector. The tests are evaluated individually, in combination with each other and especially as the dimensions of a logistic regression classifier. In the end, we conclude that, all things considered, repetition is the test that should be used.

Chapter 5 documents the covert channel detector that was built. More specifically, this chapter describes how a covert channel detector was built by using an FPGA to tap Ethernet cables and analyse the repetitiveness IP Identification values, achieving an accuracy of $98.6 \%$ with a 50 ns latency.

\subsection{Further Work}

Naturally, the research and development presented herein will not be the final word on storage covert channel detection, nor should it be. More work is needed in the future and the purpose of this section is to point to some avenues by which this thesis may be of use.

\subsubsection{More Complex Covert Channels}

The SEACCOW detection system currently operates only on relatively simple covert channels. At present it looks only at IP packets' Identification field. By design, extending the system to detect covert channels that use another field exclusively is straightforward, but a covert channel could make use of more than one field. Detecting a covert channel like that should be possible with a system similar to SEACCOW, but getting there would involve non-obvious design choices requiring further research. 


\subsubsection{Countermeasures To Evade Detection}

A countermeasure against SEACCOW could be as conceptually simple as a covert channel that uses multiple header fields, but more interestingly the way information is embedded could be altered such that it has less effect on the repetitiveness of a field. Targeted countermeasures against SEACCOW's mechanism could be of interest for continued research.

\subsubsection{Long Term Memory}

SEACCOW treats each window entirely separately from the other. Its process for identifying covert information in any given window has no memory of what happened in previous windows. Clearly, the presence of a covert message in one window is informative as to its presence in adjacent windows - recall that each window contains only 4-bytes of message. Given this, it stands to reason that this information could be used to improve the detector in some way.

\subsubsection{Generic Network Traffic Analysis}

SEACCOW was envisioned as a covert channel detection system, but most of its modules have functionality that is not specific to covert channels. By simply replacing the Repetition module, a totally different kind of analysis can be performed on some protocol-header field. Though this seems like something that would already exist as

open-source hardware, nothing adequate could be found for SEACCOW. Everything 
beyond the Ethernet cores was implemented from scratch and this work could be used by a researcher wishing to analyse protocol-headers with low latency for a purpose wholly unrelated to covert channels.

\subsubsection{More Sophisticated Test Combination}

A significant part of this thesis was focused on characterizing a variety of statistical tests applied to storage covert channels. That investigation ultimately concluded that combining of tests was not efficient for a logistic regression detector. However, other types of detector such as a decision trees or random forests could potentially make good use of test combination.

\subsection{Final Remarks}

Detecting covert channels is a challenge that seems likely to increase in importance in

the future. As organizations improve the security of their computer systems, the use of covert channels becomes more attractive to attackers because the consequent barrier to detection will outweigh the added complexity. Good covert channel detection systems ought to be fast enough to allow responders to take action and accurate enough that they will do so with confidence. 


\section{Appendix A}

\section{Covert Message}

IT is a truth universally acknowledged, that a single man in possession of a good fortune must be in want of a wife. However little known the feelings or views of such a man may be on his first entering a neighbourhood, this truth is so well fixed in the minds of the surrounding families, that he is considered as the rightful property of some one or other of their daughters. "My dear Mr. Bennet," said his lady to him one day, "have you heard that Netherfield Park is let at last?" Mr. Bennet replied that he had not. "But it is," returned she; "for Mrs. Long has just been here, and she told me all about it." Mr. Bennet made no answer. "Do not you want to know who has taken it?" cried his wife impatiently. "You want to tell me, and I have no objection to hearing it." This was invitation enough. "Why, my dear, you must know, Mrs. Long says that Netherfield is taken by a young man of large fortune from the north of England; that he came 
down on Monday in a chaise and four to see the place, and was so much delighted with it that he agreed with Mr. Morris immediately; that he is to take possession before Michaelmas, and some of his servants are to be in the house by the end of next week." "What is his name?" "Bingley." "Is he married or single?" "Oh! single, my dear, to be sure! A single man of large fortune; four or five thousand a year. What a fine thing for our girls!" "How so? how can it affect them?" "My dear Mr. Bennet," replied his wife, "how can you be so tiresome! You must know that I am thinking of his marrying one of them." "Is that his design in settling here?" "Design! nonsense, how can you talk so! But it is very likely that he may fall in love with one of them, and therefore you must visit him as soon as he comes." "I see no occasion for that. You and the girls may go, or you may send them by themselves, which perhaps will be still better; for, as you are as handsome as any of them, Mr. Bingley might like you the best of the party." "My dear, you flatter me. I certainly have had my share of beauty, but I do not pretend to be any thing extraordinary now. When a woman has five grown up daughters, she ought to give over thinking of her own beauty." "In such cases, a woman has not often much beauty to think of." "But, my dear, you must indeed go and see Mr. Bingley when he comes into the neighbourhood." 


\section{Appendix B}

\section{Code Repositories}

This thesis is accompanied by two code repositories that are made publicly available.

Experiment Source Code: gitlab.com/CyberSEA-Public/CCStatTests

SEACCOW Source Code: gitlab.com/CyberSEA-Public/SEACCOW 


\section{Bibliography}

[Alt16] Altera. Cyclone IV Device Handbook, 2.1 edition, December 2016.

[BGC05] Vincent Berk, Annarita Giani, and George Cybenko. Detection of covert channel encoding in network packet delays. Technical Report TR2005-536, Dartmouth College, Hanover, NH, U.S.A., August 2005.

[CAC95] Gregory J Chaitin, Asat Arslanov, and Cristian Calude. Program-size complexity computes the halting problem. Technical report, Department of Computer Science, The University of Auckland, New Zealand, 1995.

[CBS04] Serdar Cabuk, Carla E. Brodley, and Clay Shields. IP covert timing channels: design and detection. In Proceedings of the 11th ACM conference on Computer and communications security, pages 178-187. ACM, 2004.

[CBS09] Serdar Cabuk, Carla E. Brodley, and Clay Shields. IP covert channel detection. ACM Transactions on Information and System Security (TISSEC), 12(4):22, 2009. 
[CCG13] Valentino Crespi, George Cybenko, and Annarita Giani. Engineering statistical behaviors for attacking and defending covert channels. IEEE Journal of Selected Topics in Signal Processing, 7(1):124-136, February 2013.

[Col05] Lasse Collin. A quick benchmark: Gzip vs. bzip2 vs. lzma. Web site: http: //tukaani.org/lzma/benchmarks. html [Last accessed: 22 October 2019], 2005.

[Dod08] Yadolah Dodge. The concise encyclopedia of statistics. Springer Science \& Business Media, 2008.

[For01] Lance Fortnow. Kolmogorov complexity. In Aspects of Complexity (Short Courses in Complexity from the New Zealand Mathematical Research Institute Summer 2000 Meeting, Kaikoura), volume 4, pages 73-86, 2001.

[GAOG16] Ross K. Gegan, Vishal Ahuja, John D. Owens, and Dipak Ghosal. Realtime gpu-based timing channel detection using entropy. In 2016 IEEE Conference on Communications and Network Security (CNS), pages 296305. IEEE, 2016.

[Gar17] Sebastian Garcia. Normal captures. https://stratosphereips.org, 2017. Malware Capture Facility Project.

[GBC05] Annarita Giani, Vincent Berk, and George Cybenko. Covert channel detection using process query systems. In Proceedings of the 2nd Annual Conference for Network Flow Analysis, FLOCON 2005, September 2005. 
[GW10] Steven Gianvecchio and Haining Wang. An entropy-based approach to detecting covert timing channels. IEEE Transactions on Dependable and Secure Computing, 8(6):785-797, 2010.

[GZ17a] Hendra Gunadi and Sebastian Zander. Bro covert channel detection (BroCCaDe) framework: Design and implementation. Technical Report 20171117B, Murdoch University, 2017.

[GZ17b] Hendra Gunadi and Sebastian Zander. Bro covert channel detection (BroCCaDe) framework: Scope and background. Technical Report 20171117A, Murdoch University, 2017.

[GZ17c] Hendra Gunadi and Sebastian Zander. Extending bro covert channel detection (BroCCaDe) with new plugins. Technical Report 20171207A, Murdoch University, 2017.

[GZ18] Hendra Gunadi and Sebastian Zander. Performance evaluation of the bro covert channel detection (BroCCaDe) framework. Technical Report 20180427A, Murdoch University, 2018.

[Ins18] Ponemon Institute. 2018 cost of a data breach study: Global overview. Technical report, IBM Security, 2018.

[Int19] Intel. Triple-Speed Ethernet Intel FPGA IP User Guide, 17.1 edition, January 2019.

[Jas10] Jason Jaskolka. Modeling, analysis, and detection of information leakage via protocol-based covert channels. Master's thesis, McMaster University, 2010. 
[JK11] Mamatha V Jadhav and Suvarna L Kattimani. Effective detection mechanism for TCP based hybrid covert channels in secure communication. In Proceedings of the 2011 International Conference on Emerging Trends in Electrical and Computer Technology, ICETECT 2011, pages 1123-1128, March 2011.

[JKZ12] Jason Jaskolka, Ridha Khedri, and Qinglei Zhang. On the necessary conditions for covert channel existence: A state-of-the-art survey. Procedia Computer Science, 10:458-465, 2012.

[KL51] Solomon Kullback and Richard A. Leibler. On information and sufficiency. Annals of Mathematical Statistics, 22(1):79-86, 1951.

[Lam73] Butler W Lampson. A note on the confinement problem. Communications of the ACM, 16(10):613-615, 1973.

[LCL $\left.{ }^{+} 03\right]$ Ming Li, Xin Chen, Xin Li, Bin Ma, and Paul Vitányi. The similarity metric. In Proceedings of the fourteenth annual ACM-SIAM symposium on Discrete algorithms, pages 863-872. Society for Industrial and Applied Mathematics, 2003.

[LCW $\left.{ }^{+} 15\right]$ Anyi Liu, Jim X. Chen, Harry Wechsler, et al. Real-time timing channel detection in an software-defined networking virtual environment. Intelligent Information Management, 7(06):283, 2015.

[LCY11] Anyi Liu, Jim Chen, and Li Yang. Real-time detection of covert channels in highly virtualized environments. In International Conference on Critical Infrastructure Protection, pages 151-164. Springer, 2011. 
[LZ76] Abraham Lempel and Jacoblem Ziv. On the complexity of finite sequences. IEEE Transactions on information theory, 22(1):75-81, 1976.

[LZCF17] Qingbao Li, Ping Zhang, Zhifeng Chen, and Guangxin Fu. Covert timing channel detection method based on random forest algorithm. In Communication Technology (ICCT), 2017 IEEE 17th International Conference on, pages 165-171. IEEE, 2017.

[Mic06] Micron Technology, Inc. DDR3 SDRAM, 2006. Rev. P 2/12.

[NBSD12] Bukke Devendra Naik, Sarath Chandra Boddukolu, Pothula Sujatha, and P Dhavachelvan. Connecting entropy-based detection methods and entropy to detect covert timing channels. In N. Meghanathan, D. Nagamalai, and N. Chaki, editors, Proceedings of the 2nd International Conference on Advances in Computing and Information Technology, volume 176 of Advances in Intelligent Systems and Computing, pages 279-288. Springer Berlin/Heidelberg, 2012.

[Pav19] Igor Pavlov. Lzma sdk. Web site: https://www.7-zip.org/sdk.html [Last accessed: 28 April 2020], 2019.

[PBL $\left.{ }^{+} 98\right]$ Alberto Porta, Giuseppe Baselli, Diego Liberati, Nicola Montano, Chiara Cogliati, Tomaso Gnecchi-Ruscone, Alberto Malliani, and Sergio Cerutti. Measuring regularity by means of a corrected conditional entropy in sympathetic outflow. Biological cybernetics, 78(1):71-78, 1998. 
[RHS17] Fahimeh Rezaei, Michael Hempel, and Hamid Sharif. Towards a reliable detection of covert timing channels over real-time network traffic. IEEE Transactions on Dependable and Secure Computing, 14(3):249-264, 2017.

[SK06] Marc Smeets and Matthijs Koot. Covert channels. Tech. Rep.; University of Amsterdam; Amsterdam, Netherlands; 2006.

[SSM03] Taeshik Sohn, JungTaek Seo, and Jongsub Moon. A study on the covert channel detection of TCP/IP header using support vector machine. In S. Qing, D. Gollmann, and J. Zhou, editors, Information and Communications Security, volume 2836 of Lecture Notes in Computer Science, pages 313-324. Springer Berlin/Heidelberg, 2003.

[TA05] Eugene Tumoian and Maxim Anikeev. Network based detection of passive covert channels in TCP/IP. In Proceedings of the 30th IEEE Conference on Local Computer Networks, pages 802-807, Sydney, Australia, 2005.

[Ter17] Terasic Technologies. DE2-115 User Manual, 2.3 edition, 2017.

[US 85] US Department of Defense. Computer security requirements: Guidance for applying the department of defense trusted computer system evaluation criteria in specific environments. The 'Orange Book' Series, pages $557-572,1985$.

[XKC20] Jiarong Xing, Qiao Kang, and Ang Chen. NetWarden: Mitigating network covert channels while preserving performance. In 29th USENIX Security Symposium (USENIX Security 20), 2020. 
[ZL77] Jacob Ziv and Abraham Lempel. A universal algorithm for sequential data compression. IEEE Transactions on information theory, 23(3):337-343, 1977.

[ZL78] Jacob Ziv and Abraham Lempel. Compression of individual sequences via variable-rate coding. IEEE transactions on Information Theory, 24(5):530-536, 1978.

[ZLD10] Jiangtao Zhai, Guangjie Liu, and Yuewei Dai. A covert channel detection algorithm based on TCP markov model. In Proceedings of the 2nd International Conference on Multimedia Information Networking and Security, MINES 2010, pages 893-897, Los Alamitos, CA, U.S.A., 2010.

[ZS10] Hong Zhao and Yun Q. Shi. A phase-space reconstruction approach to detect covert channels in TCP/IP protocols. In Proceedings of the 2010 IEEE International Workshop on Information Forensics and Security, WIFS 2010, pages 1-6, Piscataway, NJ, U.S.A., 2010. 